\title{
Resonance Surfaces for Forced Oscillators
}

\author{
Richard P. McGehee and Bruce B. Peckham
}

\section{CONTENTS}

Introduction

1. Periodically Forced Oscillators

2. Resonance

3. Classical Local Bifurcations

4. Resonance Surfaces for the Caricature

Acknowledgments

References
This research was partially supported by NSF grants DMS-9020220 and DMS-9206957.
The study of resonances in systems such as periodically forced oscillators has traditionally focused on understanding the regions in the parameter plane where these resonances occur. Resonance regions can also be viewed as projections to the parameter plane of resonance surfaces in the four-dimensional Cartesian product of the state space with the parameter space. This paper reports on a computer study of resonance surfaces for a particular family and illustrates some advantages of viewing resonance regions in this light.

\section{INTRODUCTION}

The study of resonance regions comprises a substantial part of the recent literature on bifurcation theory. A resonance region is usually studied in the context of a two-parameter family of dynamical systems and is defined to be that set of parameter values for which the corresponding dynamical system has a certain type of periodic orbit.

Traditionally, the study of resonance has focused on understanding the regions in parameter space where certain resonances occur. These regions are usually bounded by curves that represent critical phenomena such as saddle-node bifurcations, and the computational goal has been to find ways to trace out these curves in parameter space.

On the other hand, proofs of general theorems have often used either explicitly or implicitly the fact that the resonance regions in the parameter space are projections of surfaces in the Cartesian product of the state space and the parameter space. In fact, typical continuation methods used to compute parameter space curves such as saddle-node curves actually find the curves in this larger space, then project them to the parameter plane. The curves are typically smooth along a smooth twodimensional (resonance) surface in the bigger space, 
but when projected to the parameter plane are only piecewise smooth.

This paper reports on a computer study of these resonance surfaces for a particular family of maps of the plane and attempts to illustrate the advantages of viewing resonance regions as projections from the bigger space. Although this point of view has already inspired many mathematicians, it has been difficult to actually visualize the surfaces. Modern technology has played a significant role in our work by allowing us to visualize our numerical experiments and the surfaces to which the experiments led us. While we think the illustrations in this paper are more illuminating than traditional parameter space bifurcation diagrams, we note that it is vastly more enlightening to watch the surfaces as they are interactively rotated in the four-dimensional space and projected to the screen of a computer graphics workstation.

Surface visualization has led us to a deeper understanding of certain bifurcation phenomena and their interrelations. For example, the classification of the classical local bifurcations presented in Section 3 was inspired by the computer visualization.

The surface emphasis may also affect future computational goals for numerical bifurcation studies. The resonance surfaces in this paper were much easier to compute than were the bifurcation curves along the surfaces. Being able to project the surfaces to the parameter space makes it less imperative to compute the bifurcation curves, which often just project to the edges of the resonance regions. We hope our work will provide deeper understanding of bifurcation theory and inspiration for future work.

\section{PERIODICALLY FORCED OSCILLATORS}

\subsection{The Differential Equation Model}

Differential equations that can be classified as periodically forced planar oscillators abound in science and engineering. A standard model is

$$
\dot{x}=V(x)+\alpha W(x, \omega t),
$$

where the dependent variable $x$ is a point in the Euclidean plane $\mathbb{R}^{2}$ and where the dot represents the derivative with respect to the independent variable $t$. The real parameters $\alpha$ and $\omega$ are the forcing amplitude and the forcing frequency. The function $W$ is periodic in its second variable with period one. Both $V$ and $W$ are assumed to be $C^{\infty}$.

The solutions of (1.1) of interest in this paper are the resonant or entrained solutions, namely those that are periodic with period an integer multiple of the forcing period. The forcing period for (1.1) is $\omega^{-1}$, so we say that a solution of (1.1) is in resonance if it has period $q / \omega$, where $q$ is a positive integer.

The basic problem addressed in this paper is that of describing the resonance regions, that is, the set of parameter values for which resonance occurs. The literature containing pictures of resonance regions is extensive. Representative pictures appear, for example, in the following very incomplete list: [Kai and Tomita 1979; Kevrekidis et al. 1986; Schrieber et al. 1988; Vance and Ross 1989; Frouzakis et al. 1991]. Figure 1 shows computergenerated pictures of some of these resonance regions for a system described below as a caricature of a forced oscillator.

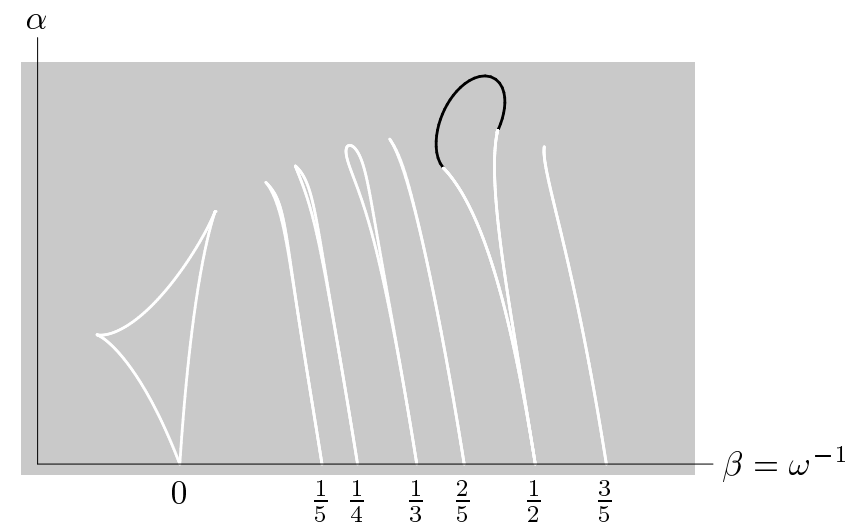

FIGURE 1. Resonance regions in parameter space for the family $H_{\mu}$ of Section 2.3. The leftmost region has $q=1$. For the remaining regions, $q$ is the denominator of the rational number at which the region is rooted. The boundary of the region $q=2$ is composed of two qualitatively different pieces; see table at bottom of page 232 . 
When $\alpha=0,(1.1)$ reduces to the planar vector field

$$
\dot{x}=V(x) .
$$

We assume this equation to have an equilibrium point $c_{0}$ inside a periodic orbit $C_{0}$ that has frequency $\omega_{0}>0$, and hence period $\omega_{0}^{-1}$. We also assume the equilibrium point to be repelling, and the periodic orbit to have characteristic multiplier strictly less than one: that is, $C_{0}$ is a normally hyperbolic attracting periodic orbit. We call $C_{0}$ the unforced oscillator of the system, and $\omega_{0}$ the natural frequency. For simplicity we assume that the unforced flow travels counterclockwise around $C_{0}$ and that $C_{0}$ is globally attracting, that is, every solution except the unstable equilibrium point $c_{0}$ asymptotically approaches $C_{0}$ as $t \rightarrow \infty$, as shown in Figure 2.

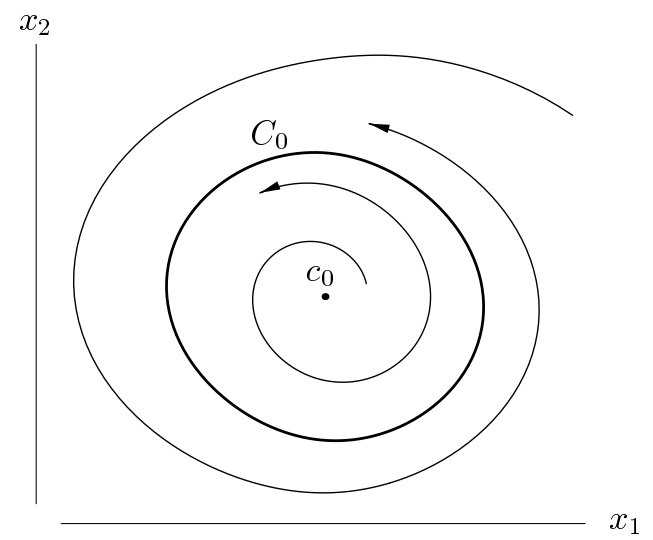

FIGURE 2. Qualitative behavior assumed of the unforced oscillator.

\subsection{The Stroboscopic Family}

It is convenient to introduce the ratio of the natural frequency and the forcing frequency,

$$
\beta:=\omega_{0} / \omega,
$$

and to use it as the first parameter instead of $\omega$. This allows consideration of cases where the natural frequency $\omega_{0}$ is zero or negative and eliminates only the case when $\omega$ is zero, which corresponds to time-independent forcing; from now on we exclude this case, assuming $\omega$ to be positive.
It is also convenient to assume that $\alpha$ is nonnegative and to combine the two parameters into a single symbol

$$
\mu:=(\beta, \alpha) \in P:=\mathbb{R} \times[0, \infty) .
$$

The stroboscopic family is obtained from (1.1) by following solutions for one period of the forcing term. More precisely, let $\varphi_{(\beta, \alpha)}(\xi, t)$ be the solution of (1.1) with initial value $x=\xi$ at $t=0$. The stroboscopic maps of the plane are defined by

$$
f_{(\beta, \alpha)}(\xi):=\varphi_{(\beta, \alpha)}\left(\xi, \omega^{-1}\right) .
$$

For each $\mu \in P$, the map $f_{\mu}$ is a diffeomorphism of the plane and is as smooth as the original equation (1.1), which, in this paper, is assumed to be $C^{\infty}$.

Remark. The stroboscopic maps carry all the essential dynamical information about (1.1). In particular, $\xi$ is a fixed point of $f_{\mu}$ if and only if $\varphi_{\mu}(\xi, t)$ is a periodic solution of (1.1) with period $1 / \omega$, and $\xi$ is a periodic point of $f_{\mu}$ with period $q$ if and only if $\varphi_{\mu}(\xi, t)$ is a periodic solution of (1.1) with period $q / \omega$. The stability properties of invariant sets of solutions of (1.1) are reflected by the stability properties of the corresponding invariant sets for the map $f_{\mu}$.

When $\alpha=0$, each stroboscopic map reduces to the time $1 / \omega$ map of the autonomous equation (1.2). The equilibrium point $c_{0}$ becomes a repelling fixed point for the map, while the periodic orbit $C_{0}$ becomes a normally hyperbolic attracting invariant circle on which the map is conjugate to a rigid rotation. This invariant circle is globally attracting in the sense that it is asymptotically approached by the iterates of every point except $c_{0}$.

The rotation number of the unforced oscillator plays a key role in the analysis. Recall that the rotation number of an orientation-preserving homeomorphism of the circle is defined (modulo 1) as the asymptotic average angular increase, in units of $2 \pi$ radians; in particular, for a rigid rotation of a circle through $2 \pi \rho$ radians, the rotation number is $\rho$. For the unforced oscillator, the rotation number 
of the map $f_{(\beta, 0)}$ restricted to the invariant circle $C_{0}$ is $\beta$.

In this paper we use the term "generic" loosely; roughly speaking, a property is "generic" if it occurs for a topologically large set of maps or vector fields for some appropriate topology on the space of maps or vector fields. For $\alpha>0$, the family $f_{\mu}$ is assumed to be generic in the space of $C^{\infty}$ two-parameter families of diffeomorphisms of the plane. It is important to note that the family $f_{\mu}$ is far from generic if $\alpha=0$ is included in the parameter space. In particular, when restricted to the invariant circle $C_{0}$, the map $f_{(\beta, 0)}$ is conjugate to a rigid rotation through an angle of $2 \pi \beta$ radians. For example, if $\beta$ equals the rational number $p / q$, every point on the circle $C_{0}$ is periodic with period $q$. Thus the family of stroboscopic maps of a "generic" family of forced oscillators will necessarily contain "nongeneric" maps corresponding to zero forcing amplitude.

\subsection{A Caricature}

This paper is a computer study of a particular family of maps. Instead of picking a differential equation of the form (1.1) and numerically integrating the equation to arrive at the stroboscopic maps, we chose instead to pick an easily computable family of diffeomorphisms having the features of the family of stroboscopic maps generated by a family of forced oscillators. This choice avoided the numerical problems introduced by an integration method and allowed us to achieve computations of great accuracy and detail without relying on massive computer resources.

The family of maps $H_{(\beta, \alpha)}$ is defined as the composition

$$
H_{(\beta, \alpha)}:=g_{\alpha} \circ h_{\beta},
$$

where $g_{\alpha}$ and $h_{\beta}$ are defined as follows.

For $\beta \in \mathbb{R}$, the map $h_{\beta}$ is taken to be the timeone map of the following planar vector field, written here in polar coordinates:

$$
\dot{r}=\frac{r\left(1-r^{2}\right)}{1+r^{2}}, \quad \dot{\theta}=2 \pi \beta+\frac{1-r^{2}}{1+r^{2}} .
$$

It is easy to check that this vector field satisfies the hypotheses stated above for (1.2). Indeed, the origin is a repelling equilibrium point surrounded by a hyperbolic attracting periodic orbit on the unit circle. The flow restricted to the unit circle is rigid rotation through the angle $2 \pi \beta t$. Thus, for the time-one map $h_{\beta}$, the unit circle is an attracting invariant circle on which the map has rotation number $\beta$.

For $\alpha \in[0,1)$, the map $g_{\alpha}$ is defined by

$$
g_{\alpha}(z)=(1-\alpha)(z-1)+1,
$$

where $z$ is a complex number used as a coordinate on $\mathbb{R}^{2}$. Thus $g_{0}$ is the identity map, while, for $0<\alpha<1$, the map $g_{\alpha}$ is a linear contraction centered at $z=1$.

For $\alpha=0$, the map $H_{(\beta, \alpha)}$ is just the time-one map of the vector field (1.3). Thus the unit circle is an invariant circle on which the map is a rigid rotation with rotation number $\beta$. For small but positive $\alpha$, the circle distorts but remains invariant. For $\alpha$ close to 1 , the circle, and indeed all periodic orbits, have disappeared and there remains only an attracting fixed point near $z=1$. This last property is typical of many forced oscillator systems: for large forcing amplitude, the system assumes a globally attracting periodic orbit with the same frequency as the forcing term.

\section{RESONANCE}

It is clear from the remark in Section 1.2 that a point in the state space of the system (1.1) is in resonance if and only if it is a periodic point of the stroboscopic map $f_{\mu}$. It will be important to distinguish periodic points according to their least period, that is, the minimum number of iterations of the map needed for the point to return.

The order of a resonance is the least period of the periodic point. The $q$-th order resonance region, denoted $R(q)$, is the set of all parameter values $\mu$ for which $f_{\mu}$ has a periodic point of least period $q$. In general, $R(q)$ will have many different 
components, at least as many as there are distinct rational rotation numbers with denominator $q$.

\subsection{Resonance Surfaces}

The main purpose of this paper is to present the case that a good way to understand the resonance regions is to think in terms of resonance surfaces, two-dimensional submanifolds of the four-dimensional Cartesian product of the state space $\mathbb{R}^{2}$ with the parameter space $P$. More precisely, define the period-q variety $\Sigma(q)$ as

$$
\Sigma(q):=\left\{(x, \mu) \in \mathbb{R}^{2} \times P: f_{\mu}^{q}(x)=x\right\},
$$

and the least-period-q surface $\Gamma(q)$ by

$$
\Gamma(q):=\Sigma(q) \backslash \bigcup_{k=1}^{q-1} \Sigma(k)
$$

Thus $\Sigma(q)$ is the set of all points $(x, \mu)$ such that $x$ is a periodic point of $f_{\mu}$ with period $q$. Some of the points in $\Sigma(q)$ might be fixed points or periodic points of periods that divide $q$. However, these points are removed from $\Sigma(q)$ when we pass to $\Gamma(q)$, as this latter set consists only of periodic points of least period $q$. The resonance region $R(q)$ is the projection of $\Gamma(q)$ onto the parameter space.

The implicit function theorem can be used to show that, for each fixed $q \geq 1$, and for an open dense subset of the space of $C^{\infty}$ two-parameter families of forced oscillators, $\Gamma(q)$ is a $C^{\infty}$ twodimensional orientable submanifold of $\mathbb{R}^{2} \times P$. Similar arguments have been given previously [Peckham 1990]. This result justifies the statement that $\Gamma(q)$ is "generically" a smooth surface, and, for the remainder of this paper, it will be assumed that $f_{\mu}$ satisfies this property. We do not know whether the caricature family $H_{\mu}$ has this generic property, but, so far, all the resonance surfaces we have explored with the computer appear to be smooth.

Recall that for $\alpha=0$ the invariant circle $C_{0}$ is conjugate to a rigid rotation with rotation number $\beta$. If $\beta=p / q$ is rational and reduced, then $C_{0}$ consists entirely of periodic points of least period $q$, which implies that

$$
C_{0} \times\{(p / q, 0)\} \subset \Gamma(q)
$$

Since the forcing amplitude is taken to be nonnegative, $C_{0} \times\{(p / q, 0)\}$ forms part of the boundary of $\Gamma(q)$.

Note that, for any given period $q$, this gives several boundary components, one for each rotation number $p / q$. Indeed, $\Gamma(q)$ decomposes into connected components characterized by a generalization of the notion of rotation number [Peckham 1988; 1990]. For the purposes of this paper, it will suffice to consider only components that can be labeled by their boundary at $\alpha=0$. More precisely, we define $\Gamma_{p / q}$ as the connected component of $\Gamma(q)$ containing $C_{0} \times\{(p / q, 0)\}$, where, as elsewhere in this paper, the rational number is assumed to be written in lowest terms. It can be shown that, for distinct rational numbers $\beta$ and $\beta^{\prime}$, the surfaces $\Gamma_{\beta}$ and $\Gamma_{\beta^{\prime}}$ are disjoint [Peckham 1988; 1990].

\subsection{Small Forcing Amplitude}

For small forcing amplitude $\alpha_{0}$, the part of the surface $\Gamma_{p / q}$ extending from $C_{0} \times\{(p / q, 0)\}$ and restricted to $\alpha \in\left[0, \alpha_{0}\right]$ is simply an annulus. This can be seen as follows.

Since the invariant circle $C_{0}$ is normally hyperbolic for $\alpha=0$, it perturbs to a nearby invariant circle $C_{(\beta, \alpha)}$ for sufficiently small $\alpha$. Under a suitable choice of coordinates $(r, \theta)$ on the state space $\mathbb{R}^{2}$, this perturbed invariant circle can be written as the graph of a function from $\theta$ to $r$. Indeed, the entire family of invariant circles can be written as the graph of a function from $(\theta, \beta, \alpha)$ to $r$. In this way, the problem of finding periodic orbits for the stroboscopic family can be reduced to the corresponding problem for a family of circle maps, a problem that is well-studied.

When $\alpha=0$ the map on the invariant circle $C_{0}$ is conjugate to a rigid rotation with rotation number $\beta$. Therefore the angular variable $\theta$ can be chosen 
so that the stroboscopic map $f_{(\beta, \alpha)}$ restricted to $C_{(\beta, \alpha)}$ can be written

$$
\psi_{(\beta, \alpha)}(\theta)=\theta+2 \pi \beta+\alpha \gamma_{(\beta, \alpha)}(\theta) .
$$

Now let $\beta=p / q+\varepsilon$, where $\varepsilon$ is a small parameter. The $q$-th iterate of the map restricted to the invariant circle can then be written

$$
\psi_{(\beta, \alpha)}^{q}(\theta)=\theta+2 \pi p+2 \pi q \varepsilon+\alpha \gamma(\theta)+O^{2}(\varepsilon, \alpha),
$$

where $O^{2}(\varepsilon, \alpha)$ denotes terms of at least second order in $\varepsilon$ and $\alpha$, and where

$$
\gamma(\theta)=\sum_{k=0}^{q-1} \gamma_{(p / q, 0)}(\theta+2 \pi k p / q) .
$$

Now the condition that the point $\theta$ on the invariant circle is periodic with rotation number $p / q$ can be written

$$
\psi_{(\beta, \alpha)}^{q}(\theta)=\theta+2 \pi p,
$$

and therefore as

$$
2 \pi q \varepsilon+\alpha \gamma(\theta)+O^{2}(\varepsilon, \alpha)=0 .
$$

Using the implicit function theorem, one can solve this last expresson for $\varepsilon$ as a function of $\theta$ and $\alpha$. Since $r$ was already written as a function of $\beta, \alpha$, and $\theta$, the resonance surface $\Gamma_{p / q}$ has been written as the graph of a function from the variables $\theta$ and $\alpha$ to the variables $r$ and $\beta$. Thus $\Gamma_{p / q}$ is an annulus for small enough $\alpha$. It turns out that, even though the invariant circles typically lose smoothness as the forcing amplitude $\alpha$ is increased, this annulus of periodic points, being part a $C^{\infty}$ surface, is itself $C^{\infty}$ [McGehee and Peckham 1995].

Figure 3 illustrates this fact, and also the generic property that the projection of the resonance surface $\Gamma_{p / q}$ to the parameter plane is a wedge-shaped region with nonempty interior [Hall 1984]. This part of the resonance region $R(q)$ is often called an "Arnold tongue" or "Arnold horn". One such tongue emerges from every rational point on the $\beta$-axis.

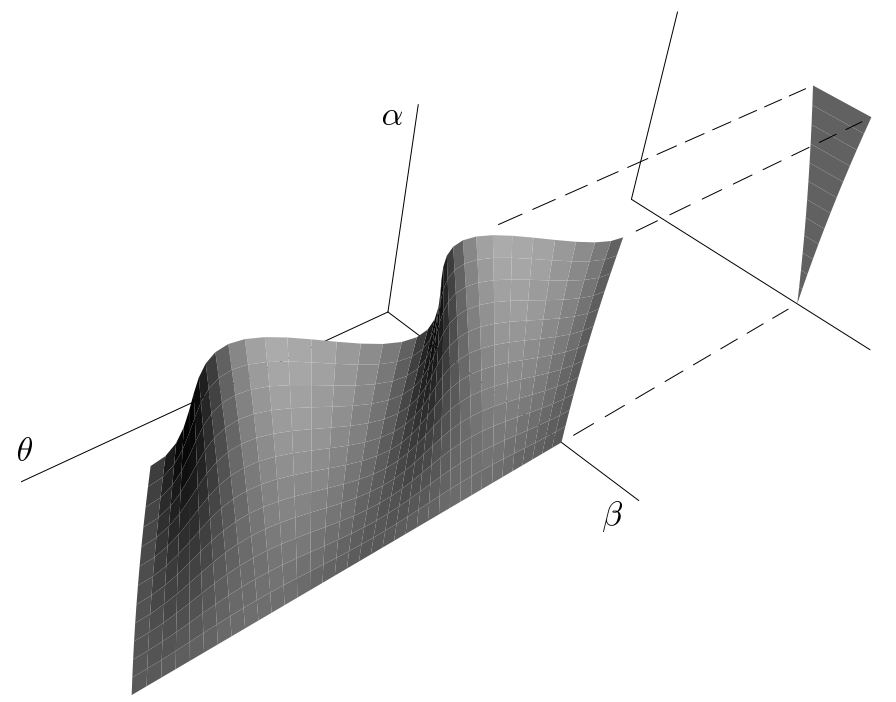

FIGURE 3. Portion of a resonance surface $\Gamma_{p / q}$ near $\alpha=0$, and its projection to the $(\beta, \alpha)$-plane. The $r$-coordinate is omitted, that is, the surface is rendered as a physical object in the three-dimensional space $(\theta, \beta, \alpha)$.

\subsection{Global Properties}

As seen above, the surfaces $\Gamma_{p / q}$ are generically two-dimensional $C^{\infty}$ orientable submanifolds of the product $\mathbb{R}^{2} \times P$. We also mentioned that, in many forced oscillator systems, large forcing amplitude produces a globally attracting periodic orbit with the same period as the forcing term, that is, a globally attracting fixed point for the stroboscopic map. It is therefore reasonable to add as a hypothesis that, for $q \neq 1$, each $\Gamma_{p / q}$ is a bounded subset of $\mathbb{R}^{2} \times P$.

Although $\Gamma_{p / q}$ is bounded, it is, in general, not closed. Indeed, the boundary of $\Gamma_{p / q}$ contains some of the standard bifurcation points. It is useful to add these bifurcation points to the surface. We therefore consider the topological closure of the resonance surface $\Gamma_{p / q}$, and denote it by $\bar{\Gamma}_{p / q}$.

A generalization of the rotation number was used previously [Peckham 1990] to show that, since $p$ and $q$ are relatively prime, the only possible closure points are fixed points, that is,

$$
\bar{\Gamma}_{p / q} \backslash \Gamma_{p / q} \subset \Sigma(1) .
$$


For $q=1$, the surface $\Gamma_{p / q}$ is closed, so this statement is vacuous. For $q \geq 2$, the closure points correspond to a fixed point bifurcating to a period- $q$ orbit.

For $q=2$, this bifurcation is referred to as a period-doubling bifurcation, and for $q \geq 3$ as a Hopf bifurcation. Generically, a Hopf bifurcation with a specified rotation number is a codimensiontwo phenomenon, which means that it occurs at an isolated point in the two-dimensional parameter space. For $q \geq 3$, therefore, $\bar{\Gamma}_{p / q} \backslash \Gamma_{p / q}$ consists of isolated points; it is exactly one point for most examples we have seen. Adding this point compactifies the surface, but the new surface is no longer $C^{\infty}$. An analysis of the normal form at a point of Hopf bifurcation shows that the surface $\bar{\Gamma}_{p / q}$ is generically only $C^{q-3}$.

Generically, a period-doubling bifurcation is a codimension-one phenomenon, which, in the case of two-parameter families, means that the bifurcation points occur along a curve in the parameter space. In most known examples, this appears to be a single circle. The open surface $\Gamma_{p / 2}$ is joined to itself along this circle to form a nonorientable surface $\bar{\Gamma}_{p / 2}$. Although orientatibility is lost after the closure operation, smoothness is generically retained.

This discussion is summarized by the following previously proved theorem [McGehee and Peckham 1995; Peckham 1990]:

Theorem. The following properties are generic for two-parameter families of forced oscillators.

1. If $q \leq 2$, then $\bar{\Gamma}_{p / q}$ is a $C^{\infty}$ submanifold of $\mathbb{R}^{2} \times$ $P$.

2. If $q \geq 3$, then $\bar{\Gamma}_{p / q}$ is a $C^{q-3}$ submanifold of $\mathbb{R}^{2} \times P$.

3. If $q \neq 2$, then $\bar{\Gamma}_{p / q}$ is orientable.

We conjecture that assuming $\Gamma_{p / 2}$ bounded implies that $\bar{\Gamma}_{p / 2}$ is generically nonorientable. It does seem that $\bar{\Gamma}_{p / 2}$ is nonorientable for the examples we have investigated. These surfaces are described and illustrated in Section 4 for the caricature family.
The numerics for that family provide strong evidence that $\Gamma_{p / q}$ is a Möbius band for $q=2$, and a disk for $q=3,4,5$.

\subsection{An Example of Closure Causing Nonorientability}

It is interesting to digress momentarily to an easily describable example that illustrates how closure can turn an orientable surface into a nonorientable one. The topology and geometry of this example closely parallel that of the period-two variety $\Sigma(2)$, which we illustrate for the caricature family later in the paper. The digression also serves to illustrate techniques used to visualize all the resonance surfaces.

Identify $\mathbb{R}^{4}$ with $\mathbb{C}^{2}$, and consider the real twodimensional variety

$$
\Sigma:=\left\{(z, w) \in \mathbb{C}^{2}: z=\bar{z} w\right\}
$$

Write $\Sigma=\Sigma_{1} \cup \Sigma_{2}$, where $\Sigma_{1}$ is the real plane (complex line) $z=0$, and $\Sigma_{2}$ is the complement. Both $\Sigma_{1}$ and $\Sigma_{2}$ are orientable real surfaces; $\Sigma_{1}$ is topologically a plane, while $\Sigma_{2}$ is homeomorphic to an open annulus, parametrized by the punctured $z$-plane. However, the closure of $\Sigma_{2}$ can be written

$$
\bar{\Sigma}_{2}=\left\{\left(r e^{i \theta}, e^{i 2 \theta}\right) \in \mathbb{C}^{2}: \theta \in[0,2 \pi), r \in[0, \infty)\right\},
$$

which can be recognized as a Möbius band and hence is nonorientable. Note that $\Sigma_{1} \cap \bar{\Sigma}_{2}=\bar{\Sigma}_{2} \backslash \Sigma_{2}$ is the circle

$$
S:=\left\{(z, w) \in \mathbb{C}^{2}: z=0,|w|=1\right\}
$$

which corresponds to $r=0$ in the parametrization of $\bar{\Sigma}_{2}$ just given; $S$ is also the subset of points $\bar{\Sigma}_{2}$ covered twice by that parametrization.

In comparing the variety $\Sigma$ of this example with the variety $\Sigma(2)$ of the caricature family, or more generally with the stroboscopic forced oscillator maps, we see that $\Sigma_{1}$ is analogous to the fixedpoint surface $\Gamma(1)$, while $\bar{\Sigma}_{2}$ is analogous to one of the period-two resonance surfaces $\bar{\Gamma}_{p / 2}$. 
Now consider only the bounded portion of $\bar{\Sigma}_{2}$ for which $|z| \leq \frac{1}{2}$ :

$$
\bar{\Sigma}_{2}^{\prime}=\left\{\left(r e^{i \theta}, e^{i 2 \theta}\right) \in \mathbb{C}^{2}: \theta \in[0,2 \pi), r \in\left[0, \frac{1}{2}\right]\right\} .
$$

Figure 4 shows the projection of $\bar{\Sigma}_{2}^{\prime}$ to the $z$-plane. This projection is, of course, just the disk $\{z \in \mathbb{C}$ : $\left.|z| \leq \frac{1}{2}\right\}$. Note, however, that the disk has variations in shading, so it appears to be something other than simply a flat disk in the plane. This appearance results from the way we are visualizing the surface $\bar{\Sigma}_{2}^{\prime}$ as a subset of $\mathbb{R}^{4}$. The surface is first projected into $\mathbb{R}^{3}$ along the imaginary $w$-axis, yielding a two-dimensional surface in $\mathbb{R}^{3}$. This surface is then rendered using the three-dimensional viewing software Geomview [Phillips et al. 1993].

More precisely, use $(\operatorname{Re} z, \operatorname{Im} z, \operatorname{Re} w, \operatorname{Im} w)$ as coordinates on $\mathbb{R}^{4}$, and use $\left(x_{1}, x_{2}, x_{3}\right)$ as coordinates on $\mathbb{R}^{3}$. The surface $\bar{\Sigma}_{2}$ is projected from $\mathbb{R}^{4}$ to $\mathbb{R}^{3}$ using the projection whose matrix in these coordinates is given by

$$
\left[\begin{array}{llll}
1 & 0 & 0 & 0 \\
0 & 1 & 0 & 0 \\
0 & 0 & 1 & 0
\end{array}\right] .
$$

Once projected into $\mathbb{R}^{3}$, the surface is then visualized as though the viewer were infinitely far out along the positive $x_{3}$-axis (looking through an infinitely powerful telescope) with the positive $x_{1}$ axis pointing to the viewer's right and the positive $x_{2}$-axis pointing up. In other words, the projection to the printed page is given by the matrix

$$
\left[\begin{array}{lll}
1 & 0 & 0 \\
0 & 1 & 0
\end{array}\right]
$$

We stress that the two projections, first from $\mathbb{R}^{4}$ to $\mathbb{R}^{3}$ and then from $\mathbb{R}^{3}$ to $\mathbb{R}^{2}$, are treated in fundamentally different ways. The surface as it sits in $\mathbb{R}^{4}$ is treated as an abstract mathematical object, endowed with no color or other attribute. In the projection from $\mathbb{R}^{4}$ to $\mathbb{R}^{3}$ one dimension's worth of information is completely lost. By contrast, the projection from $\mathbb{R}^{3}$ to $\mathbb{R}^{2}$ should be thought of as a description of the way that a photograph of the surface in $\mathbb{R}^{3}$ is taken; color, reflectivity, lights and

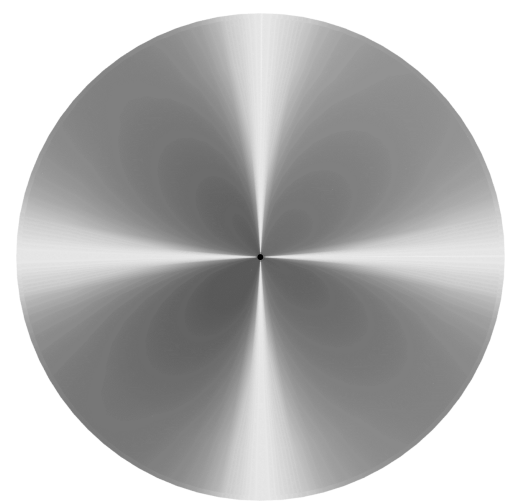

FIGURE 4. Portion of the surface $\bar{\Sigma}_{2}$ with $|z| \leq \frac{1}{2}$, projected to and rendered in the three-dimensional space $(\operatorname{Re} z, \operatorname{Im} z, \operatorname{Re} w)$, from the point at infinity in the positive $(\operatorname{Re} w)$-direction.

shadows are all used in an attempt to display three dimension's worth of information as intelligibly as possible in two dimensions.

As an illustration of this, consider Figure 5. The same projection from $\mathbb{R}^{4}$ to $\mathbb{R}^{3}$ is used there as in Figure 4. In Figure 5, however, the object has been rotated in $\mathbb{R}^{3}$ in such a way so the projection from $\mathbb{R}^{3}$ to $\mathbb{R}^{2}$ is no longer along a coordinate axis; it happens to be given by

$$
\left[\begin{array}{rrr}
0.609 & -0.622 & 0.493 \\
-0.750 & -0.250 & 0.612
\end{array}\right]
$$

Now the black dot in the center of the disc in Figure 4 can be seen to be a line. This line is the projection of the circle $S$ described above along which

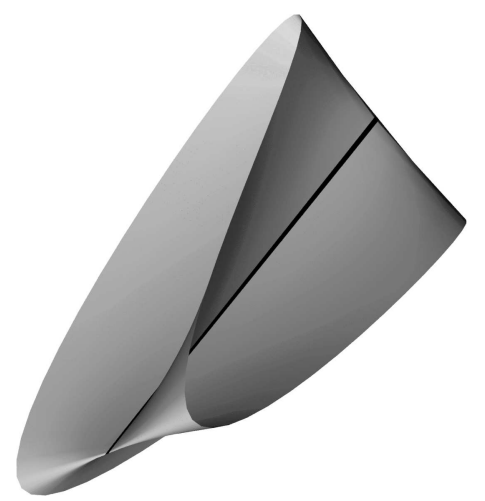

FIGURE 5. Another view of the object of Figure 4, with the same projection onto three dimensions. 
the annulus $\Sigma_{2}$ is glued to form the Möbius band $\bar{\Sigma}_{2}$. This circle is given by the conditions $z=0$ and $|w|=1$, and thus projects to the line segment in $\mathbb{R}^{3}$ given by $\operatorname{Re} z=\operatorname{Im} z=0,|\operatorname{Re} w| \leq 1$.

$S$ can be seen clearly as a circle in Figure 6, where it is again shown in black. There we have chosen a different projection from $\mathbb{R}^{4}$ to $\mathbb{R}^{3}$, given by the matrix

$$
\left[\begin{array}{llll}
0.707 & 0 & 0 & -0.707 \\
0 & 0.707 & -0.707 & 0 \\
0.5 & 0.5 & 0.5 & 0.5
\end{array}\right] .
$$

One can also think in terms of rotating $\bar{\Sigma}_{2}^{\prime}$ in $\mathbb{R}^{4}$ before projecting.

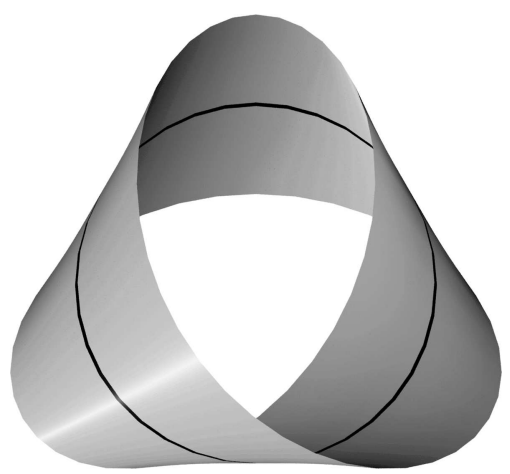

FIGURE 6. Another view of the surface $\bar{\Sigma}_{2}^{\prime}$ of Figure 4, with a different projection onto three dimensions. Here it is clear that $\bar{\Sigma}_{2}^{\prime}$ is a Möbius band; this fact was obscured in Figures 4 and 5, mainly because the projection $\mathbb{R}^{4} \rightarrow \mathbb{R}^{3}$ used there takes $\bar{\Sigma}_{2}^{\prime}$ to a nonembedded surface in $\mathbb{R}^{3}$.

\section{CLASSICAL LOCAL BIFURCATIONS}

The description and characterization of local bifurcations typically includes dynamical information, but it turns out that the local bifurcations of codimension one and two that occur in generic twoparameter families of maps of $\mathbb{R}^{2}$ can all be characterized using only singularities of the period- $q$ varieties $\Sigma(q)$ defined above and the projection of these varieties to the parameter plane. This geometric viewpoint leads to the following three natural categories of bifurcations:
1. those that can be locally characterized solely by singularities of one or more of the period- $q$ varieties;

2. those that have no nontrivial topology (no nearby variety singularities) but can be characterized by the preferred projection of a variety to parameter space; and

3. those that can be locally characterized only by considering both variety singularities and the projection of the varieties to parameter space.

We proceed to describe the classical local bifurcations of codimension one and two that occur in two parameter families of maps of $\mathbb{R}^{2}$, classifying them into these categories.

\subsection{Variety Singularities}

A singular point on the variety $\Sigma(q)$ is by definition a point in $\mathbb{R}^{2} \times P$ where the $2 \times 4$ Jacobian matrix of the defining implicit equation $f_{\mu}^{q}(x)=x$ has rank less than two. As discussed in Section 2.3, the closed resonance surface $\bar{\Gamma}_{p / q}$ has singularities generically only at the closure points $\bar{\Gamma}_{p / q} \backslash \Gamma_{p / q}$, which can occur only on the fixed-point surface $\Sigma(1)$. It follows also that these fixed points are singular points on the variety $\Sigma(q)$. Since $e^{i 2 \pi p / q}$ must be an eigenvalue of $D f_{\mu}(x)$ at these fixed points [Peckham 1990], such a bifurcation will generically be a Hopf bifurcation if $q \geq 3$, and a period-doubling bifurcation if $q=2$.

Hopf Bifurcations. A Hopf bifurcation point is a point $(x, \mu)$ on $\Gamma(1)$ at which $D f_{\mu}(x)$ has eigenvalues $e^{ \pm i 2 \pi \rho}$, where $0<\rho<\frac{1}{2}$, and for which certain nondegeneracy conditions hold. An important special case is a $p / q$-resonant Hopf bifurcation, at which $\rho=p / q$, this rational number being expressed in lowest terms. In this case, $(x, \mu)$ is a point on $\Sigma(q)$ for which $D f_{\mu}^{q}(x)$ is the identity. This singular point on the variety $\Sigma(q)$ lies both on the $C^{\infty}$ surface $\Gamma(1)$ and on the $C^{q-3}$ surface $\bar{\Gamma}_{p / q}$.

All Hopf bifurcation points occur along a curve in the surface $\Gamma(1)$, characterized by the eigenvalue condition given above. For a generic family, the points along the curve where the eigenvalues are 
roots of unity form a dense set in the Hopf bifurcation curve; clearly, the same can be said of the points of intersection of $\bar{\Gamma}_{p / q}$ and $\Gamma(1)$. So the entire curve of Hopf bifurcations can be distinguished topologically, without regard to the projection to the parameter space of any of the resonance surfaces or varieties. Look ahead to Figure 17.

When viewed as a point on the surface $\Gamma(1)$, a Hopf bifurcation point is ordinary (nonsingular). When viewed as a point on the period- $q$ surface $\bar{\Gamma}_{p / q}$, however, a resonant Hopf bifurcation point is a point where the $C^{\infty}$ smoothness of the surface breaks down; it is generically only $C^{q-3}$. This can be seen from the normal form of a $p / q$-resonant Hopf bifurcation point. A standard approach is to approximate the $q$-th iterate of the family of maps with the flow of a family of differential equations that is equivariant with respect to rotations by $p / q$ [Arnol'd 1982; Takens 1974]. The normal form of the flow is

$$
\dot{z}=\varepsilon z+A z^{2} \bar{z}+B \bar{z}^{q-1}
$$

where $z$ is a complex state space variable, $\varepsilon$ is a complex parameter, and $A$ and $B$ are complex constants. Fixed points of the original maps all correspond to $z=0$, so period- $q$ surface points are the nonzero solutions to $\dot{z}=0$. The closure of the period- $q$ surface adds back the Hopf point: $(z, \varepsilon)=(0,0)$. In polar coordinates $z=r e^{i \theta}$, the closure of the period- $q$ surface becomes

$$
\varepsilon=-A r^{2}-B r^{q-2} e^{-q i \theta} .
$$

With some recollection from multivariable calculus, the second term on the right can be shown to cause the surface to be $C^{q-3}$ but not $C^{q-2}$ at the origin. Look ahead to Figure 14, where an example of a period- 3 surface is shown near a $\frac{1}{3}$-resonant Hopf point. The surface does indeed appear to have a singularity where it is $C^{0}$ but not $C^{1}$ (last two views on bottom row).

Period Doubling. A period-doubling point is a point $(x, \mu)$ on $\Gamma(1)$ for which -1 is an eigenvalue of
$D f_{\mu}(x)$ and for which certain nondeneracy conditions hold. Thus $(x, \mu)$ is a point on $\Sigma(2)$ for which 1 is an eigenvalue of $D f_{\mu}^{2}(x)$ and hence is, as before, a singular point of the variety $\Sigma(2)$. However, in this case, the singular points occur along a curve of intersection between the two $C^{\infty}$ surfaces $\Gamma(1)$ and $\bar{\Gamma}_{p / 2}$ that make up $\Sigma(q)$. This curve of singular points topologically characterizes a perioddoubling point.

Note that the loss of smoothness that occurs on $\bar{\Gamma}_{p / q}$ for $q \geq 3$ is replaced instead by a loss of orientability of the surface $\bar{\Gamma}_{p / 2}$, as discussed in Section 2.4.

Double Negative-One Points. For a generic family, a Hopf bifurcation curve can terminate only when the eigenvalues of the linearization are both 1 or are both -1 . An alternate characterization of the double negative-one point is that, on the fixed-point surface, a Hopf bifurcation curve terminates on a period-doubling curve [Arnol'd 1982; Takens 1974]. Since both period-doubling curves and Hopf curves are characterized topologically, so is this intersection point of the two curves.

Takens-Bogdanov Points (see [Takens 1974; Bogdanov 1976]). The other possible termination point of a Hopf bifurcation curve is characterized by a double eigenvalue of 1 . An alternate interpretation is that, on the fixed-point surface $\Gamma(1)$, the Hopf bifurcation curve terminates on a curve of saddle-nodes. The Hopf curve can be located topologically. Although the saddle-node curve can be "seen" only through the geometric projection to the parameter space, a Takens-Bogdanov point can be described as a Hopf curve that terminates and has no other nearby variety singularities. The absence of other variety singuarities is what distinguishes this bifurcation from the double negative-one bifurcation.

\subsection{Projection Singularities}

All points near a nonsingular point on $\Sigma(q)$ have the same period, so it suffices to restrict attention to the resonance surfaces $\Gamma_{p / q}$. Projection singularities occur at points where the projection of $\Gamma_{p / q}$ 
to the parameter space is singular but for which, unlike the variety singularities above, the surface is otherwise topologically uninteresting. As is true for variety singularities, projection singularities occur at points $(x, \mu)$ on the surface $\Gamma_{p / q}$ where the surface fails to be locally the graph of a function from the parameter space to the state space, which implies that the linear map $D f_{\mu}^{q}(x)$-id is singular. This last condition is equivalent to the condition that 1 occurs as an eigenvalue of $D f_{\mu}^{q}(x)$.

Folds (Saddle-nodes). The most common projection singularity is the fold, or saddle-node, which occurs on $\Gamma_{p / q}$ at points $(x, \mu)$ where 1 is a simple eigenvalue of $D f_{\mu}^{q}(x)$ and where certain higherorder nondegeneracies hold. Geometrically, $\Gamma_{p / q}$ folds back on itself in $\mathbb{R}^{4}$, so that its projection to the parameter space changes from locally having two inverse images to, at the singular point, locally having one inverse image, and finally none. The edges of the surface with respect to this distinguished projection form curves of period- $q$ saddlenodes. Note that the curves of saddle-node bifurcations are generically smooth curves on the smooth surface $\Gamma_{p / q}$, and hence have no intrinsic characterization in terms of the varieties $\Sigma(q)$. From the dynamical viewpoint, the two inverse images correspond to two period- $q$ points, one a saddle and one either a sink or a source, which come together at a single degenerate period- $q$ point (a saddle-node), and then disappear.

Cusps. The only other purely projection singularity that can occur generically for two-parameter families of diffeomorphisms on $\mathbb{R}^{2}$ is the cusp, which occurs on $\Gamma_{p / q}$ at points $(x, \mu)$ where 1 is a simple eigenvalue of $D f_{\mu}^{q}(x)$ and where a certain higherorder degeneracy occurs but where certain other nondegeneracies hold. Geometrically, two folds on $\Gamma_{p / q}$ come together and disappear. The two folds project to two curves in the parameter space that come together at a cusp point, providing the name for the singularity. Locally, within the cusped region determined by the curves, the projection map has three inverse images, while outside this region it has one. This change between one and three inverse images characterizes the cusp bifurcation for generic families.

\subsection{Combinations}

Transcritical Hopf Bifurcation. Along the Hopf bifurcation curve, one sometimes finds a point separating the so-called "subcritical" and "supercritical" bifurcations [Chenciner 1985]. Topologically this is a point on a Hopf curve; it is distinguished geometrically from other Hopf points because the side of the Hopf curve to which the resonance regions project as they emanate from the resonant Hopf points changes at the transcritical Hopf point. Although such points occur for the caricature family described above, they will not be discussed further in this paper.

Transcritical Period Doubling. As one goes along the period-doubling curve, one may find, in addition to the double negative-one points mentioned above, also points where a degeneracy occurs in the higher order terms of the map [Peckham and Kevrekidis 1991]. Such a higher-order degeneracy point is characterized topologically by living on a perioddoubling curve and is distinguished geometrically from other period-doubling points in that the local projection of the period-two surface $\Gamma(2)$ changes at these points from projecting to only one side of the period-doubling curve to projecting to both sides of the curve.

Dynamically, these points separate two types of stability characteristics of the period-two orbit bifurcating from the fixed point: on one side the bifurcating period-two orbit is a saddle, and on the other it is either a sink or a source.

\section{RESONANCE SURFACES FOR THE CARICATURE}

Recall that the the caricature family of Section 1.3 is written $H_{(\beta, \alpha)}\left(x_{1}, x_{2}\right)$, where $\left(x_{1}, x_{2}\right)$ is a point in the state space $\mathbb{R}^{2}$ and $(\beta, \alpha)$ is a point in the parameter space $P=\mathbb{R} \times[0,1)$. The parameter $\alpha \in[0,1)$ is the forcing amplitude, and the parameter $\beta \in \mathbb{R}$ is the rotation number for zero forcing 


\section{REMARKS ON THE FIGURES}

Figure 1, and all of the figures in Section 4, represent objects that naturally live in the four-dimensional product $\mathbb{R}^{2} \times P$ of the phase space and the parameter space $P$. The latter extends for $\alpha \in[0,1]$ and $\beta \in(-\infty, \infty)$. Fixed points exist for all of these parameter values, but we have restricted our fixed-point surface figures to $\alpha \in[0, .75]$ and $\beta \in[-.15,1.3]$. For $\alpha>.75$ the only periodic point is a globally attracting fixed point, while $\beta$ only appears modulo 1 in the definition of our family (1.3). Our figures thus contain all the interesting topology of periodic points in slightly more than a fundamental $\beta$-interval (see Figures 8, 9, and 17, top).

To generate the data for these surfaces, we used variations of Newton's method and standard continuation techniques. Typically, we computed enough cross sections on each surface so that we could be relatively certain of its topology and hence be able to decide on a reasonable parametrization. We were then able to model the surface in $\mathbb{R}^{4}$, project it from $\mathbb{R}^{4}$ to $\mathbb{R}^{3}$, and use the program Geomview [Phillips et al. 1993] to view the resulting surface in three dimensions. The positioning in $\mathbb{R}^{3}$ is represented by a "viewing projection" from $\mathbb{R}^{3}$ to $\mathbb{R}^{2}$. As explained in Section 2, because most of the figures are intended to be seen as three-dimensional, the two separate projections are of interest rather than just their composition as a projection from $\mathbb{R}^{4}$ to $\mathbb{R}^{2}$.

To describe the projections from four to three dimensions, let the coordinates in $\mathbb{R}^{4}$ be ordered $\left(\beta, \alpha, x_{1}, x_{2}\right)$. In each case we start by scaling $\alpha$ and $\beta$ by 10 . Next we sometimes rotate or shear the $\left(\beta, x_{2}\right)$-plane, leaving the other two coordinates fixed; finally, we drop the last coordinate. In one exceptional case we instead rotate the $\left(\alpha, x_{1}\right)$-plane and drop the third coordinate. Explicitly, each projection is one of:

$$
A(\gamma)=\left[\begin{array}{crcc}
10 \cos \pi \gamma & 0 & 0 & \sin \pi \gamma \\
0 & 10 & 0 & 0 \\
0 & 0 & 1 & 0
\end{array}\right], \quad B=\left[\begin{array}{rrll}
10 & 0 & 0 & 0.2 \\
0 & 10 & 0 & 0 \\
0 & 0 & 1 & 0
\end{array}\right], \quad C=\left[\begin{array}{clcc}
10 & 0 & 0 & 0 \\
0 & 7.07 & -0.707 & 0 \\
0 & 0 & 0 & 1
\end{array}\right] .
$$

The projections from three to two dimensions can be described in terms of modified Euler angles. We rotate by an angle $\theta$ around the first coordinate axis, then by an angle $\psi$ around the second coordinate axis, and then drop the third coordinate. The result is of the form $P(\theta, \psi)=\left[\begin{array}{ccc}\cos \psi & \sin \theta \sin \psi & \cos \theta \sin \psi \\ 0 & \cos \theta & -\sin \theta\end{array}\right]$.

Here are the projections used. An asterisk $*$ indicates the projection $\mathbb{R}^{3} \rightarrow \mathbb{R}^{2}$ is further followed by a $-90^{\circ}$ rotation in the plane of the paper.

\begin{tabular}{|l|l|l||l|l|l|}
\hline \multicolumn{1}{|c|}{ Figure(s) } & $\mathbb{R}^{4} \rightarrow \mathbb{R}^{3}$ & \multicolumn{1}{|c|}{$\mathbb{R}^{3} \rightarrow \mathbb{R}^{2}$} & \multicolumn{1}{|c|}{ Figure $(\mathrm{s})$} & $\mathbb{R}^{4} \rightarrow \mathbb{R}^{3}$ & \multicolumn{1}{c|}{$\mathbb{R}^{3} \rightarrow \mathbb{R}^{2}$} \\
\hline $1,7,10,13$, & $A(0)$ & $P\left(0^{\circ}, 0^{\circ}\right)$ & 12 (right) & $C$ & $P\left(-45^{\circ}, 30^{\circ}\right)$ \\
14 (top right), 15, & \multicolumn{2}{|c|}{ (i.e., projection to } & 14 (top left) & $B$ & $P\left(-40^{\circ}, 30^{\circ}\right)$ \\
16 (top right) & \multicolumn{2}{|c|}{ the parameter plane) } & 14 (middle right) & $A(0)$ & $P\left(-70^{\circ}, 40^{\circ}\right)$ \\
8,9 & $B$ & $P\left(-20^{\circ},-20^{\circ}\right)$ & 14 (bottom left) & $A(.5)$ & $P\left(-40^{\circ}, 30^{\circ}\right)$ \\
11 (top left) & $B$ & $P\left(-40^{\circ}, 30^{\circ}\right)$ & 14 (bottom middle) & $A(.5)$ & $P\left(50^{\circ}, 0^{\circ}\right)$ \\
11 (top/bottom right), & $A(.5)$ & $P\left(-90^{\circ}, 0^{\circ}\right)$ & 16 (top left) & $B$ & $P\left(-30^{\circ}, 30^{\circ}\right)$ \\
14 (bottom right), & (i.e., projection to & 16 (middle right) & $A(0)$ & $P\left(-60^{\circ}, 10^{\circ}\right) *$ \\
16 (bottom right) & \multicolumn{2}{|c|}{ the phase plane) } & 16 (bottom left) & $A(.05)$ & $P\left(-60^{\circ}, 40^{\circ}\right) *$ \\
11 (bottom left) & $A(0)$ & $P\left(-30^{\circ}, 53^{\circ}\right)$ & 16 (bottom middle) & $A(.2)$ & $P\left(-60^{\circ}, 40^{\circ}\right) *$ \\
11 (bottom middle) & $A(0)$ & $P\left(-30^{\circ},-45^{\circ}\right)$ & 17 (top) & $B$ & $P\left(-20^{\circ}, 5^{\circ}\right)$ \\
12 (left) & $A(0)$ & $P\left(0^{\circ},-40^{\circ}\right)$ & 17 (bottom) & $B$ & $P\left(-20^{\circ}, 20^{\circ}\right)$ \\
\hline
\end{tabular}

The curves are drawn according to the following conventions:

\begin{tabular}{|l|l|l|}
\hline \multicolumn{1}{|c|}{ Curve of } & Appearance & \multicolumn{1}{c|}{ Characterizing property } \\
\hline \hline Saddle-node bifurcations & White & $\begin{array}{l}\Gamma_{p / q} \text { projects nontransversely to the parameter space; } \\
\text { equivalently, } D H_{\mu}^{q}(x) \text { has 1 as an eigenvalue }\end{array}$ \\
\hline Hopf bifurcations & Thin black & $D H_{\mu}(x)$ has complex conjugate eigenvalues on the unit circle \\
\hline Period-doubling bifurcations & Thick black & $D H_{\mu}(x)$ has -1 as an eigenvalue \\
\hline
\end{tabular}


amplitude of the invariant circle $C_{0}$, which, in this case, is the unit circle in the $\left(x_{1}, x_{2}\right)$-plane.

The remainder of this paper is devoted to a description of computer-generated pictures of various resonance surfaces for this family. We emphasize that we have not attempted to prove that the caricature family is a generic example. However, the computations and resulting pictures are consistent with the properties expected of a generic two-parameter family as described in the sections above.

For reference, the explicit projections used for the figures are given on the facing page. It is helpful, when viewing each figure, to keep in mind what projection is being used: for this reason we have sometimes repeated this information in the figure captions.

\subsection{The Fixed-Point Surface}

Figure 7 shows the local fixed-point bifurcations for the caricature family, projected to the parameter plane. The curves can be identified by reference to the bottom table on the facing page; these conventions for their appearance will be used consistently. We now discuss each type of curve.

The white curves represent fixed-point saddlenode bifurcations, that is, points $(\beta, \alpha)$ where the Jacobian matrix for a fixed point of $H_{(\beta, \alpha)}$ has an eigenvalue of 1 . Each encloses a triangular-shaped

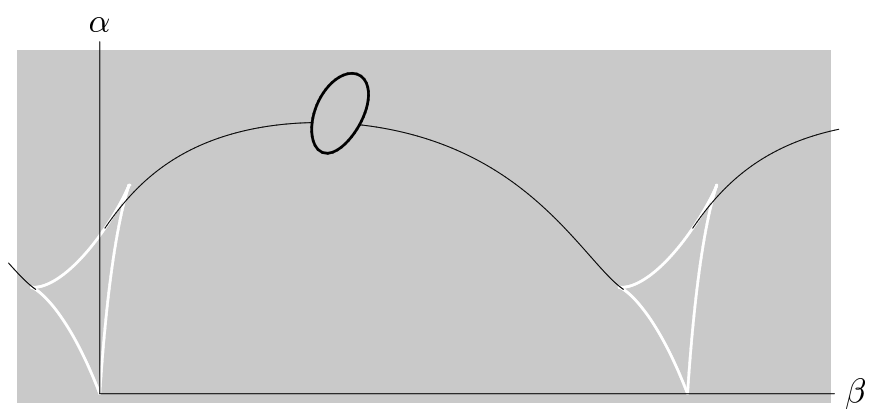

FIGURE 7. Local fixed-point bifurcations for the caricature family $H_{\mu}$, projected to the parameter plane. The curves of fixed-point saddle-node bifurcations are white, the curves of Hopf bifurcations are black and thin, and the period-doubling curve is black and thick. region with one vertex on the $\beta$-axis. For parameter points in the interior of these regions the map has three fixed points, and for parameter points outside there is only one fixed point. This means the fixed-point resonance region is the entire parameter space; the triangular regions denote parameter values whose corresponding maps having additional fixed points. The two top vertices of each triangular curve are cusp points, as described in Section 3.2, but the vertex on the $\beta$ axis is the tip of the small forcing amplitude resonance horn. This horn was described above in Section 2.2 and Figure 3; further description appears below.

The thin black curve represents the Hopf bifurcation curve, that is, the set of points $(\beta, \alpha)$ where the Jacobian matrix for a fixed point of $H_{(\beta, \alpha)}$ has a complex pair of eigenvalues on the unit circle. The thick black curve represents the period-doubling curve, that is, the set of points $(\beta, \alpha)$ where the Jacobian matrix for a fixed point of $H_{(\beta, \alpha)}$ has an eigenvalue of -1 .

Each Hopf bifurcation curve abuts at one end on a saddle-node curve and at the other end on the period-doubling curve. The point at the end abutting on a saddle-node curve is a Takens-Bogdanov point, while the point at the other end is a double negative-one point (Section 3.1).

The fixed-point surface $\Sigma(1)$ for the caricature family $H_{\mu}$ is a smooth connected surface embedded in $\mathbb{R}^{2} \times P$. A portion of it is shown in Figure 8 .

All of the $p / 1$-resonance surfaces $\Gamma_{p / 1}$, for $p \in \mathbb{Z}$, are the same, and all are equal to $\Sigma(1)$, which is diffeomorphic to the parameter space $P$ with a countable number of open discs removed. One component of the boundary of $\Sigma(1)$ consists of the repelling fixed point $c_{0}=(0,0)$ at zero forcing amplitude (recall the unforced oscillator of Figure 2), that is,

$$
\left\{\left(\left(x_{1}, x_{2}\right),(\beta, \alpha)\right) \in \mathbb{R}^{2} \times P: x_{1}=x_{2}=\alpha=0\right\} .
$$

This boundary is the bottom edge in Figure 8 . (The left, right and top edges in Figure 8 come from our having restricted the parameter space for 


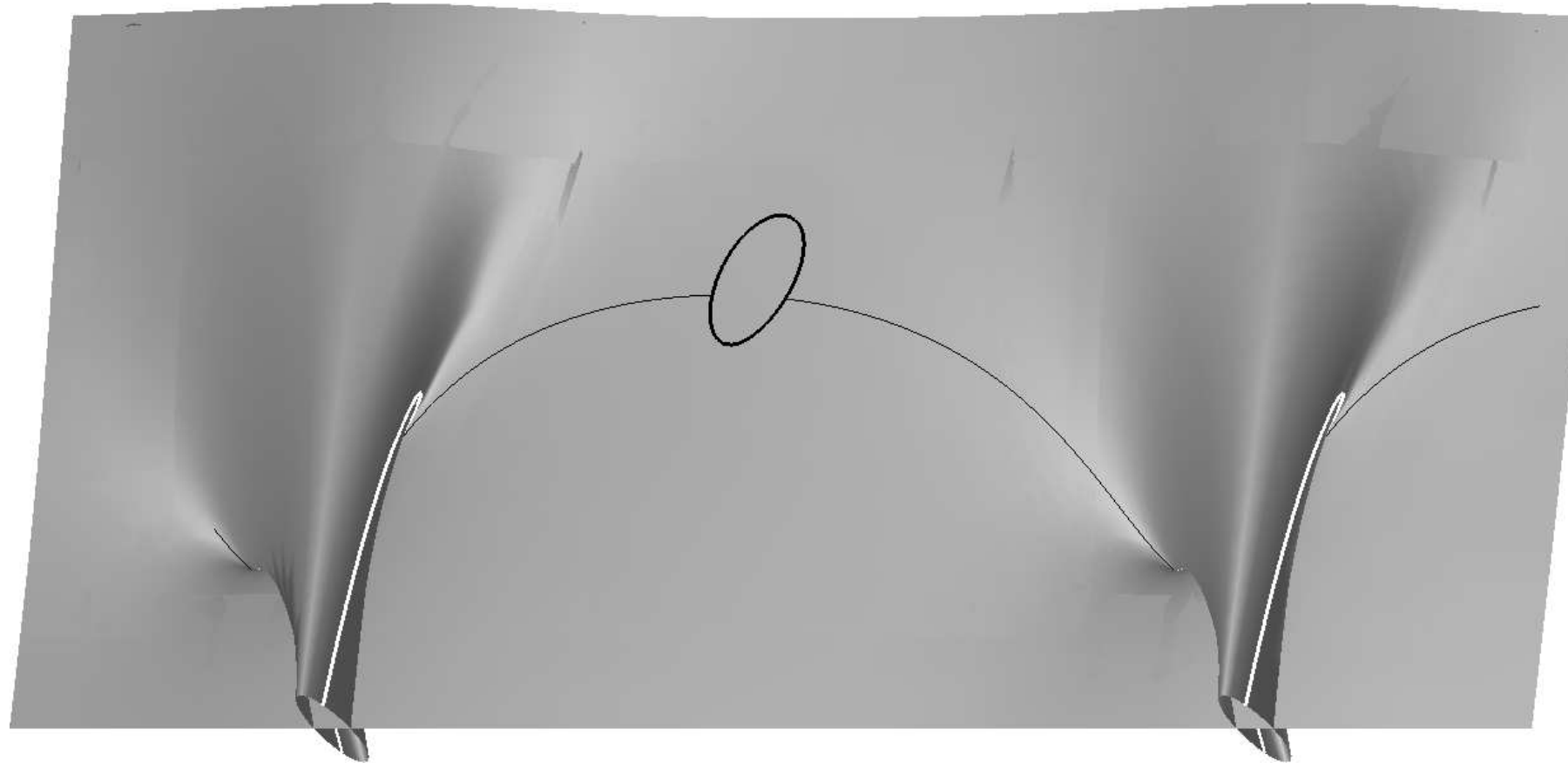

FIGURE 8. Portion of the fixed-point surface $\Sigma(1)$ for the family $H_{\mu}$, plus the curves of local bifurcation that lie on it, all projected from $\mathbb{R}^{2} \times P$ to $\mathbb{R}^{3}$ to the plane. The projections $\mathbb{R}^{4} \rightarrow \mathbb{R}^{3}$ and $\mathbb{R}^{3} \rightarrow \mathbb{R}^{2}$ differ only slightly from those of Figure 7: we have mixed a bit of $x_{2}$ with $\beta$ in projecting down to three dimensions, and the line of sight for viewing the result is not far removed from the direction of the $x_{1}$-axis. The slight discontinuities in shading are due to the piecewise way in which the surface is parametrized.

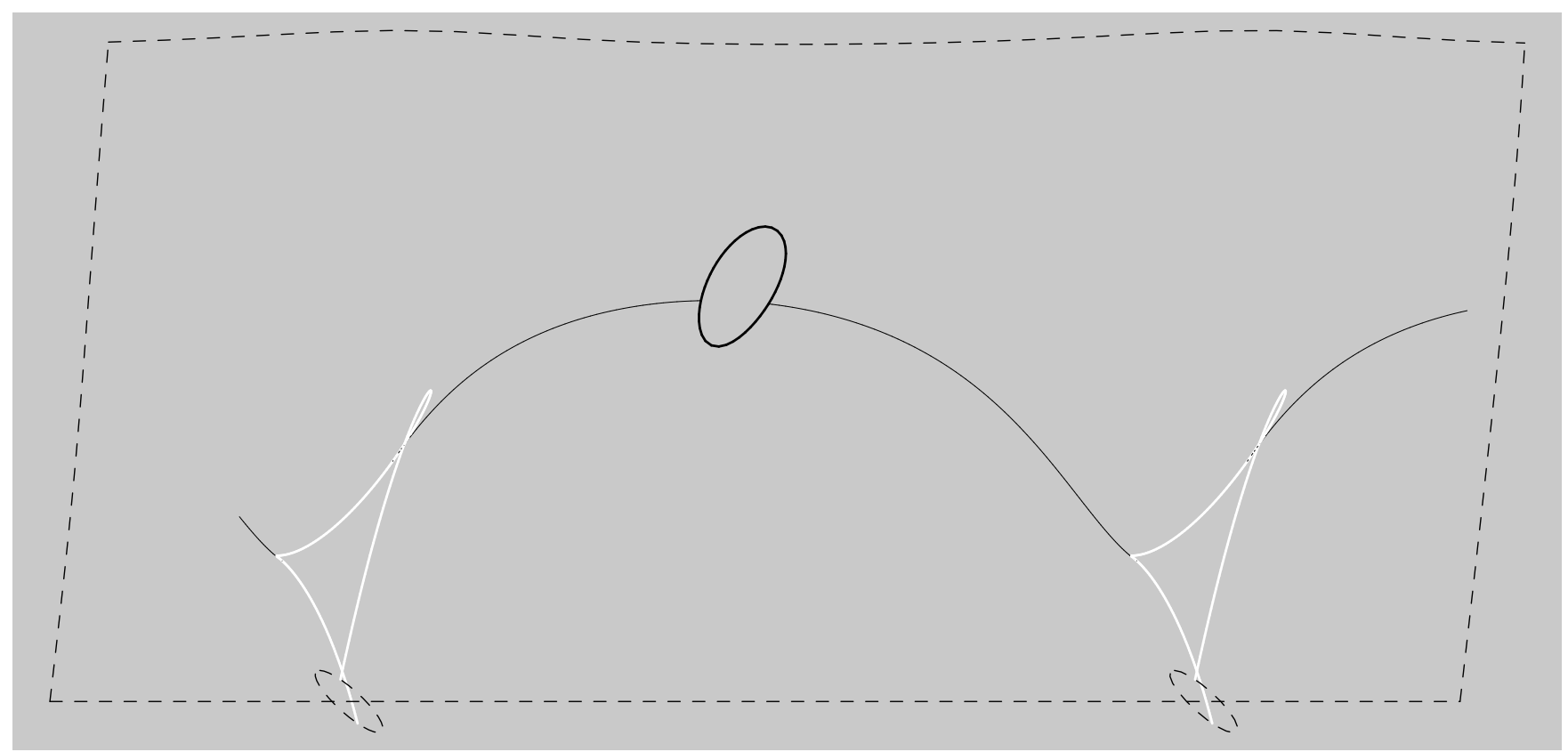

FIGURE 9. Same view as Figure 8, with the surface made transparent. 
the figure. In actuality, $\Sigma(1)$ extends to $\alpha=1$ in the positive $\alpha$ direction and infinitely both ways in the $\beta$ direction.)

Each of the remaining components of the boundary consists of the unforced invariant circle $C_{0}$ at forcing frequency equal to an integer multiple of the unforced frequency. In other words, the components are labeled by the integer $p$, and component $p$ is given by

$\left\{\left(\left(x_{1}, x_{2}\right),(\beta, \alpha)\right) \in \mathbb{R}^{2} \times P: x_{1}^{2}+x_{2}^{2}=1, \alpha=0, \beta=p\right\}$.

Figure 8 shows two of these components, for $p=0$ and $p=1$. They are the elliptical curves along the bottom edge of the surface. The figure also illustrates the fact that, near each "elliptical" boundary component, the fixed-point surface is a topological cylinder that projects to a horn-shaped region of parameter space, as described in Section 2.2. (The elliptical boundary components of Figures 8 and 9 correspond to the bottom edge of the surface in Figure 3.) Note that the elliptical boundary components intersect the linear boundary component in this projection from $\mathbb{R}^{4}$ to $\mathbb{R}^{3}$, even though they are disjoint as subsets of $\mathbb{R}^{4}$.

The saddle-node, Hopf bifurcation, and perioddoubling curves all appear in Figure 8 as they lie on the fixed-point surface. They can be seen more clearly in Figure 9, where the surface has been removed to show the parts of these curves that are hidden in Figure 8.

\subsection{The Period-Two Surface}

Figure 10 shows the resonance region corresponding to $\bar{\Gamma}_{1 / 2}$, that is, periodic points with period two and rotation number $\frac{1}{2}$. As before, the thin black curves are Hopf bifurcation curves, while the thick black curve is the curve of period-doubling. The white curves again denote saddle-nodes, in this case of period two. In other words, a point $(\beta, \alpha)$ on a white curve has the property that $H_{(\beta, \alpha)}$ has a point $\left(x_{1}, x_{2}\right)$ having least period two and such that $D H_{(\beta, \alpha)}^{2}\left(x_{1}, x_{2}\right)$ has 1 as an eigenvalue.

The union of the oval and funnel regions in Figure 10 is the projection onto the parameter space of the the period-two resonance surface $\bar{\Gamma}_{1 / 2}$. This surface, which turns out to be a Möbius band, is illustrated in Figures 11 and 12. Although it is an embedded submanifold of $\mathbb{R}^{2} \times P$, all but one of the projections from four to three dimensions shown in these figures introduce self-intersections.

The boundary of $\bar{\Gamma}_{1 / 2}$ consists of the unforced invariant circle $C_{0}$ at forcing frequency equal to half the unforced frequency, that is,

$\left\{\left(\left(x_{1}, x_{2}\right),(\beta, \alpha)\right) \in \mathbb{R}^{2} \times P: x_{1}^{2}+x_{2}^{2}=1, \alpha=0, \beta=\frac{1}{2}\right\}$.

This boundary, the base of the shape in Figure 11 (top left), projects to the parameter plane as a single point at the $\alpha=0$ tip of the $\frac{1}{2}$ resonance region of Figure 10. The top right panel shows the projection to the phase plane; compare Figure 4, where we pictured the model Möbius band.

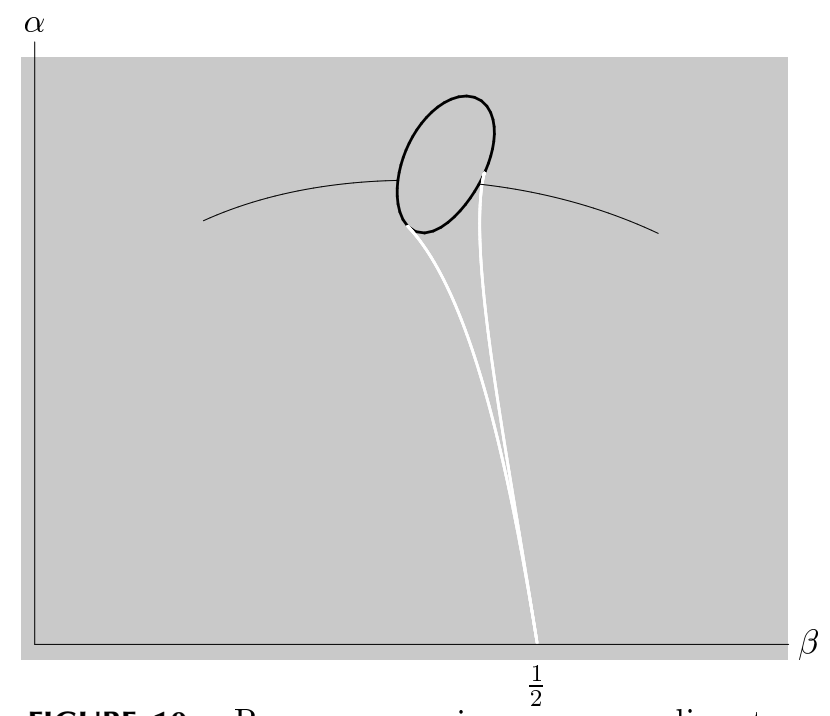

FIGURE 10. Resonance region corresponding to $\bar{\Gamma}_{1 / 2}$ (projection of the resonance surface to the parameter plane). Inside the period-doubling curve (thick black oval) there is a single period-two orbit, and inside the funnel made by the saddle-node curves (white) there are two period-two orbits, each with two points. Elsewhere there are no period-two points. The Hopf bifurcation curves (thin black) abut on the oval at the double negative-one points (Section 3.3), and the saddle-node curves abut on the oval at the points of transcritical period doubling. 


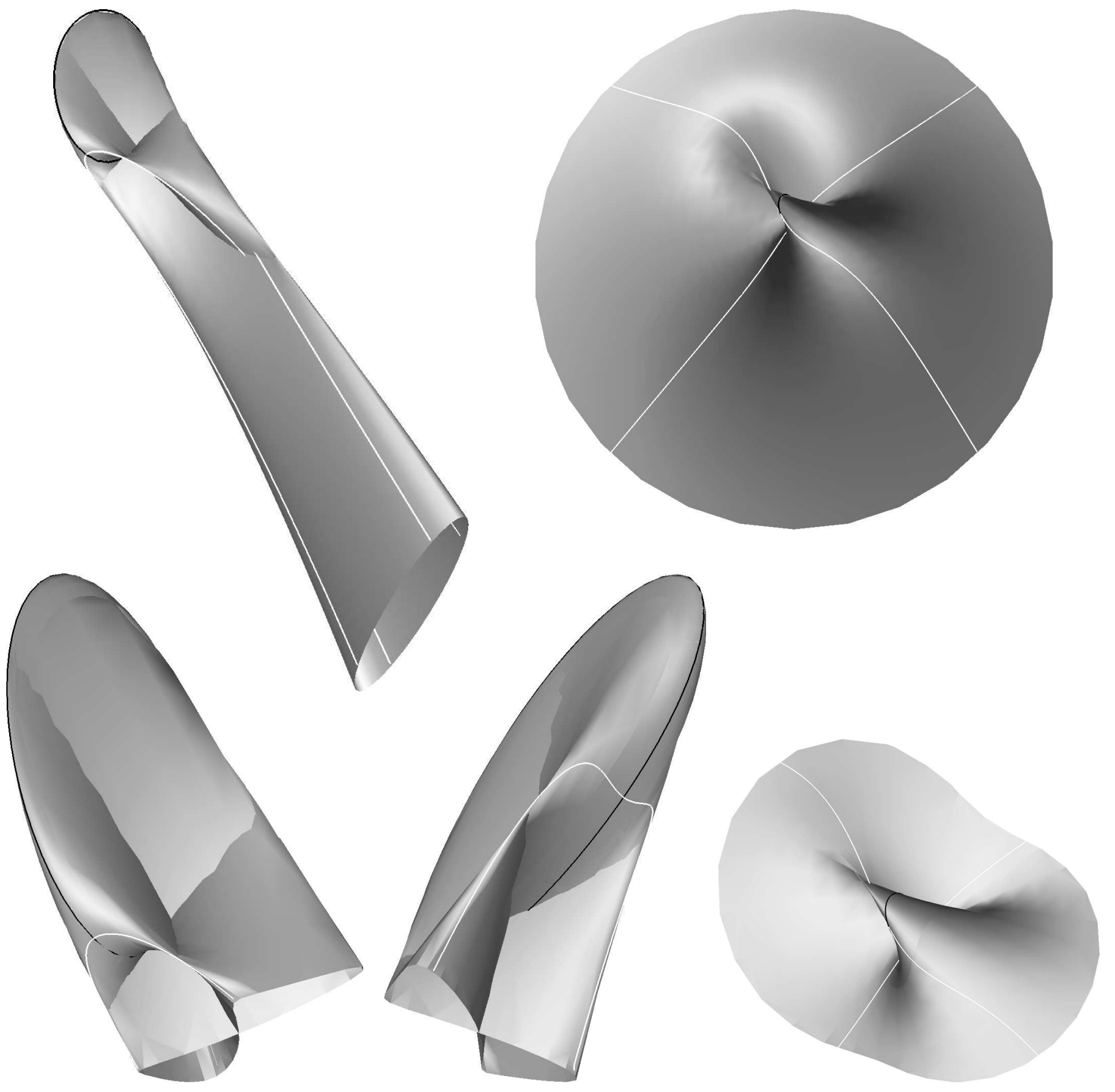

FIGURE 11. Several views of the resonance surface $\bar{\Gamma}_{1 / 2}$. Top: the whole surface, in a generic projection (left) and projected onto the phase space (right). Bottom: portion of the surface with $\alpha \geq .38$. Again the right panel shows the projection onto the phase space, and therefore is a zoom-in of the one immediately above it; compare also the view of the Möbius strip example in Figure 5. On the bottom left and middle we have the same projection $\mathbb{R}^{4} \rightarrow \mathbb{R}^{3}$ as for the top left figure, but the projections $\mathbb{R}^{3} \rightarrow \mathbb{R}^{2}$ differ. Here one sees clearly the self-intersections introduced by the projection from $\mathbb{R}^{4}$ to $\mathbb{R}^{3}$. Note in particular that the boundary curve, where the surface was arbitrarily cut for the purposes of illustration, looks like a figure eight in this projection. It is actually a topological circle in $\mathbb{R}^{4}$, as is clear in the right panel. 

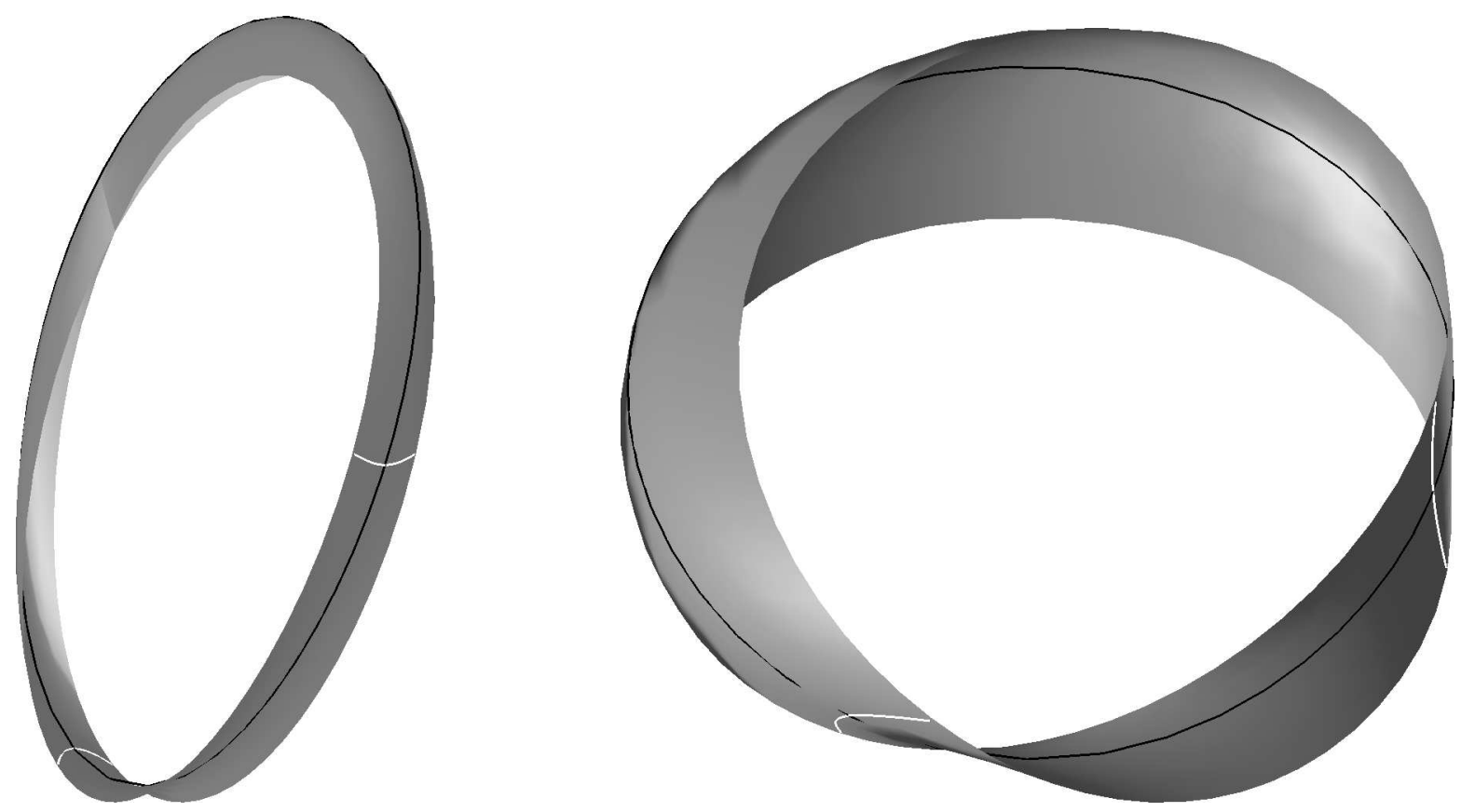

FIGURE 12. A smaller piece of $\Gamma_{1 / 2}$, around the period-doubling circle. The projection for the view on the left is somewhat similar to those of Figure 11, left. The projection on the right was chosen so that the intermediate surface in $\mathbb{R}^{3}$ is an embedded Möbius band (compare with the model Möbius band in Figure 6).

As described in Section 2.2, the portion of $\bar{\Gamma}_{1 / 2}$ restricted to $\alpha \in\left[0, \alpha_{0}\right]$ is a topological cylinder for $\alpha_{0}$ small enough. To get a better view of the interesting part of the topology of $\bar{\Gamma}_{1 / 2}$, which occurs near the thick black period-doubling curve, we have sliced it off at $\alpha=.38$. The discarded part below $\alpha=.38$ is contractible to the boundary circle, so the topology of the resulting surface is the same as for the full surface. The resulting surface is pictured in the bottom row of Figure 11.

It is interesting to note that the saddle-node curves (white) intersect the period-doubling curve (thick black) transversely on the surface $\bar{\Gamma}_{1 / 2}$, in constrast to the parameter-plane projection of Figure 10, where the saddle-node curve appears to end on the period-doubling curve. These intersection points are transcritical period-doubling points, as discussed in Section 3.3.

Figure 12 shows the surface $\bar{\Gamma}_{1 / 2}$ very near the period-doubling curve. Here the surface is clearly seen to be a Möbius band. The part of $\bar{\Gamma}_{1 / 2}$ not shown in Figure 12 is still a topological annulus (cylinder) whose outside boundary is the boundary of $\bar{\Gamma}_{1 / 2}$ (see Figure 11, top row) and whose inside boundary is identified with the boundary of the surface in Figure 12. Therefore the full surface $\bar{\Gamma}_{1 / 2}$ is also a Möbius band.

The Möbius band in Figure 12 was defined so that, as its boundary curve is traversed once, its projection to the parameter plane goes around a circle twice. This can be interpreted dynamically as follows. If one starts at a point on the Möbius band boundary and follows the boundary until its projection wraps around the parameter space circle once, the starting and ending points together form a period-two orbit.

\subsection{The Period-Three Surface}

Figure 13 shows the resonance region corresponding to $\bar{\Gamma}_{1 / 3}$, that is, periodic points with period three and with rotation number $\frac{1}{3}$. As usual, the thin black curve is a Hopf bifurcation curve, while the white curve denotes period-three saddle-nodes. 

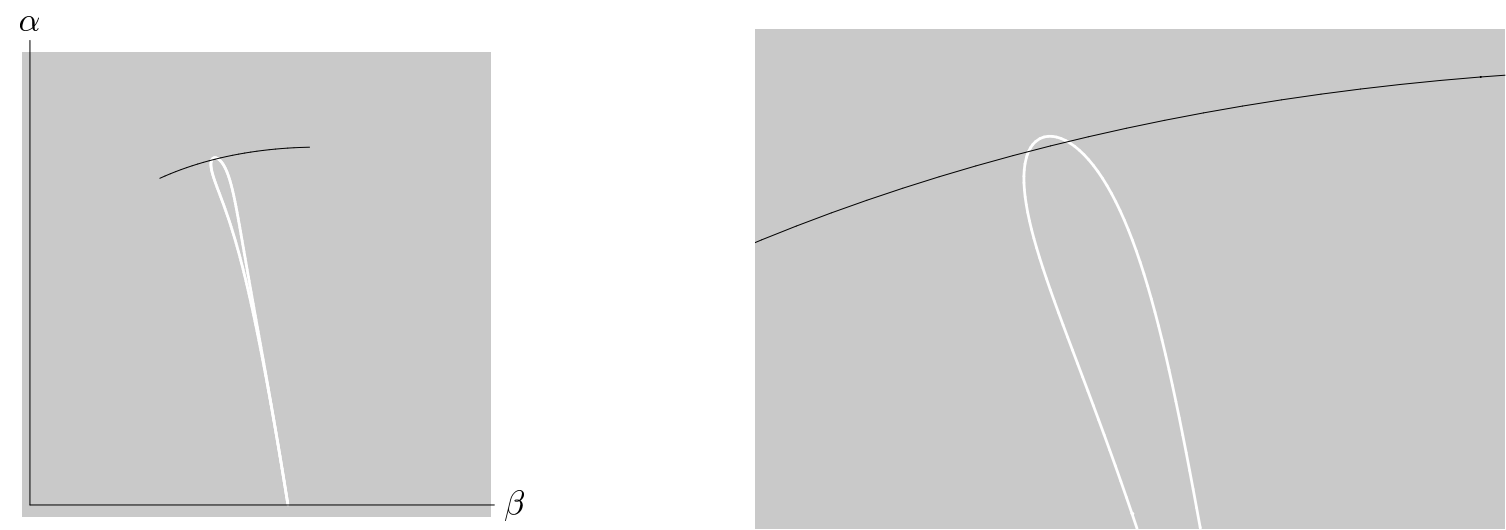

FIGURE 13. Resonance region corresponding to $\bar{\Gamma}_{1 / 3}$. Outside the loop bounded by the white curve there are no period-three points with rotation number $\frac{1}{3}$. Inside the loop there are six period-three points, divided into two orbits; one of these orbits collapses to a fixed point at the parameter value corresponding to the $\frac{1}{3}$ resonant Hopf point. The panel on the right is a blowup, showing how the Hopf bifurcation curve (black) passes through the interior of the resonance region. The $\frac{1}{3}$ resonant Hopf parameter point (not shown) lies on the Hopf curve inside the $\frac{1}{3}$ resonance region.

The blowup on the right illustrates clearly how, as predicted by the normal form [Arnol'd 1982; Takens 1974], the Hopf bifurcation point of third order projects to the interior of the resonance region. We discuss this further in Section 4.4. For higher-order resonances, namely $q \geq 5$ and sometimes $q=4$, the Hopf bifurcation point projects to the boundary.

The resonance surface corresponding to this resonance region is shown in Figure 14. As before, the ellipse forming the boundary is the invariant circle $C_{0}$, this time at the parameter point $\beta=\frac{1}{3}$, $\alpha=0$. As seen in Section 2.2, the part of $\bar{\Gamma}_{1 / 3}$ corresponding to small forcing amplitudes is a topological cylinder that projects to the horn-shaped region emanating from $\beta=\frac{1}{3}, \alpha=0$; the whole boundary circle projects to the point $\beta=\frac{1}{3}, \alpha=0$.

The projection into three dimensions shown at the top left in Figure 14 has self-intersections that might suggest some interesting topological feature, but this turns out not to be the case. This is clearly seen in the other views, where we have thrown out a cylinder contractible to the boundary circle, keeping only a portion of $\bar{\Gamma}_{1 / 3}$ near the Hopf bifurcation curve (the same for all five views). Thus $\bar{\Gamma}_{1 / 3}$ has the same topology as this portion, which is a topological disk.
Note that the shading in the last two views suggests a singular point near the center of the disk. This is the point of Hopf bifurcation; here the disk is $C^{0}$ but fails to be $C^{1}$ (recall the discussion of smoothness near Hopf points in Section 3.1). Note also that the white saddle-node lines do not intersect this point. Because the saddle-nodes project to the boundary of the $\frac{1}{3}$ resonance region, as in the top right of Figure 14, this is an illustration of the fact that the Hopf bifurcation point generically occurs in the interior of the resonance region, as mentioned above.

\subsection{The Period-Four Surface}

Figure 15 shows the resonance region corresponding to $\bar{\Gamma}_{1 / 4}$, that is, periodic points with period four and with rotation number $\frac{1}{4}$. As usual, the thin black curve is a Hopf bifurcation curve, while the white curve denotes period-four saddle-nodes.

Recall that the Hopf bifurcation point of third order generically occurs in the interior of the resonance region of period three; the Hopf point is isolated from the saddle-node curves. By contrast, all generic Hopf bifurcation points of order five and above project to the boundary of the corresponding resonance region; the Hopf point is at the common intersection of the $2 q$ saddle-node curves. The two 

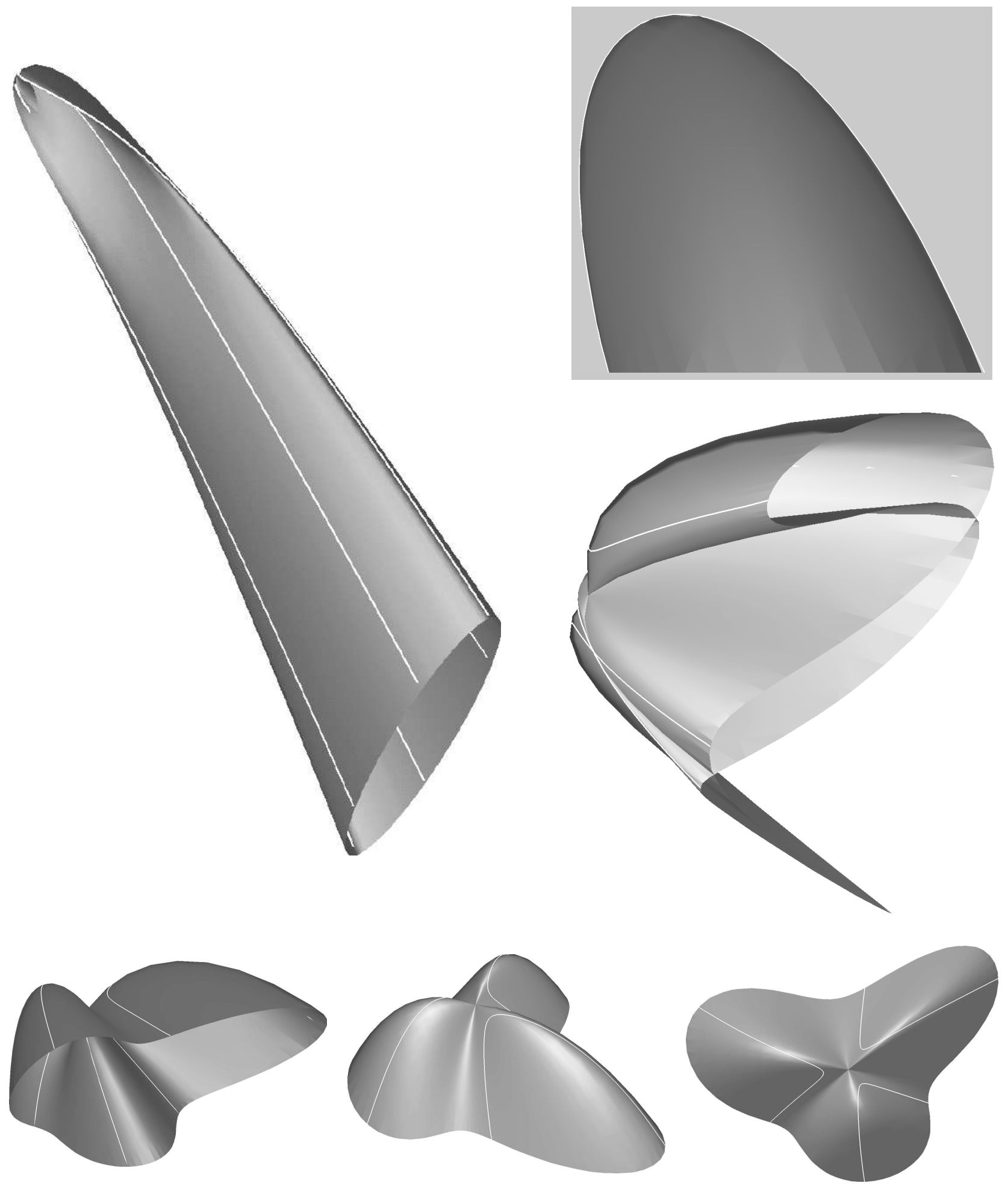

FIGURE 14. Several views of the resonance surface $\Gamma_{1 / 3}$. Top left: the whole surface, in a self-intersecting projection to three dimensions. Right and bottom: a subset of the surface near the tip, shown in various projections (top right, projection to the parameter plane; bottom right, projection to the phase plane). The complementary portion is an annulus contractible to the boundary, so the topology of $\Gamma_{1 / 3}$ is preserved, and is clearly seen to be that of a disk. 


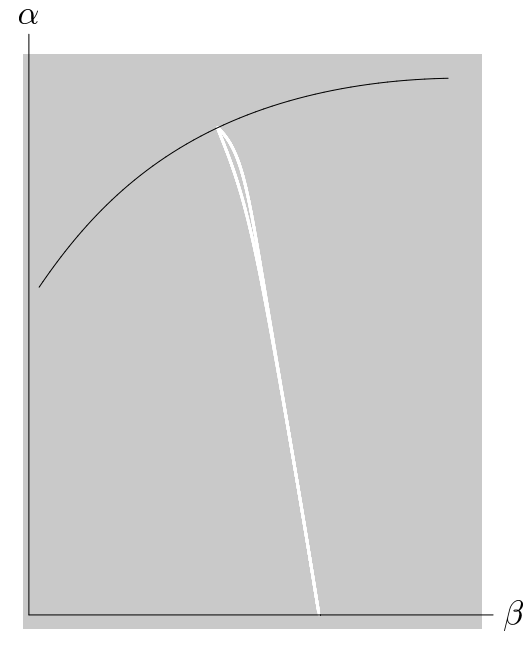

FIGURE 15. Resonance region corresponding to $\bar{\Gamma}_{1 / 4}$. Outside the white loop there are no period-three points with rotation number $\frac{1}{4}$; inside the loop there are eight such points, divided into two orbits. For this family the Hopf point is at the common intersection of the eight saddle-node curves: the black curve does not go inside the loop.

different cases can be seen directly from the normal form in (3.1). If $q=3$, the $-B r^{q-2} e^{-q i \theta}$ term dominates the $-A r^{2}$ term (for $r$ near zero), while if $q \geq 5$, the $-A r^{2}$ term dominates the $-B r^{q-2} e^{-q i \theta}$ term. By fixing $r$ and varying $\theta$ from 0 to $2 \pi$, we see that in the $q=3$ case, a circle, covered three times, is swept out around the origin in the $\varepsilon$ parameter plane; this puts the third-order Hopf point in the interior of the resonance region. For $q \leq 5$, the Hopf point is outside the circles; this puts the fifth- and higher-order Hopf points on the boundary of the corresponding resonance regions. More specifically, the Hopf points project to the tip of a resonance horn.

When $q=4$, both terms on the right-hand side of (3.1) are of the same order $r^{2}$. If $|A|<|B|$, the fourth-order resonance appears analogous to the third-order resonance; if $|A|>|B|$, the fourthorder resonance appears analogous to higher-order ones.

For the caricature family, the $\frac{1}{4}$ resonant Hopf bifurcation point appears to project to the boundary of the resonance region shown in Figure 15, analogous to the higher-order resonances. For this reason, we do not show any surfaces with periods higher than four in this paper.

The resonance surface $\bar{\Gamma}_{1 / 4}$ is shown in Figure 16 (top left). Once again, the ellipse forming the boundary is the invariant circle $C_{0}$, this time at the parameter point $\beta=\frac{1}{4}, \alpha=0$. The surface $\bar{\Gamma}_{1 / 4}$ in $\mathbb{R}^{4}$ is a disk bounded by this circle.

The remaining panels in Figure 16 show a portion of $\bar{\Gamma}_{1 / 4}$ near the Hopf bifurcation curve, again with the same topology as the full surface. That is, $\bar{\Gamma}_{1 / 4}$ is a disk. The view at the top right is the projection of this portion of the surface to the parameter plane; note that the white saddle-node curves form the boundary of the region in the parameter plane. The view in the middle right shows the same projection from $\mathbb{R}^{4}$ to $\mathbb{R}^{3}$ but a different projection from $\mathbb{R}^{3}$ to $\mathbb{R}^{2}$; we see that the intermediate surface in $\mathbb{R}^{3}$ is self-intersecting. Note also that there are eight distinct white saddle-node curves, corresponding to the existence of eight distinct period-four points for each parameter point in the interior of the resonance region.

The bottom row in Figure 16 shows other projections $\mathbb{R}^{4} \rightarrow \mathbb{R}^{3}$ of the same piece of $\bar{\Gamma}_{1 / 4}$; the last one shows most clearly that the surface is a disk. Note that the eight saddle-node curves all come together at the same point on the disk. This point is the Hopf bifurcation with eigenvalues $e^{ \pm i \pi / 2}$. Generically, the surface should be $C^{1}$ but not $C^{2}$ at this point. It does appear to be $C^{1}$, but it is hard to see whether it fails to be $C^{2}$.

One should contrast the bottom right views in Figures 14 and 16. For $\bar{\Gamma}_{1 / 4}$, the saddle-node curves all intersect at the Hopf bifurcation point, illustrating the property that the Hopf bifurcation occurs on the boundary of the resonance region in the parameter space. For the period-three surface, the saddle-node curves miss the Hopf bifurcation point, corresponding to the property that the Hopf bifurcation occurs in the interior of the resonance region. The two figures are also consistent with the generic expectation that the $\frac{1}{4}$ surface is $C^{1}$ but the $\frac{1}{3}$ surface is only $C^{0}$. 

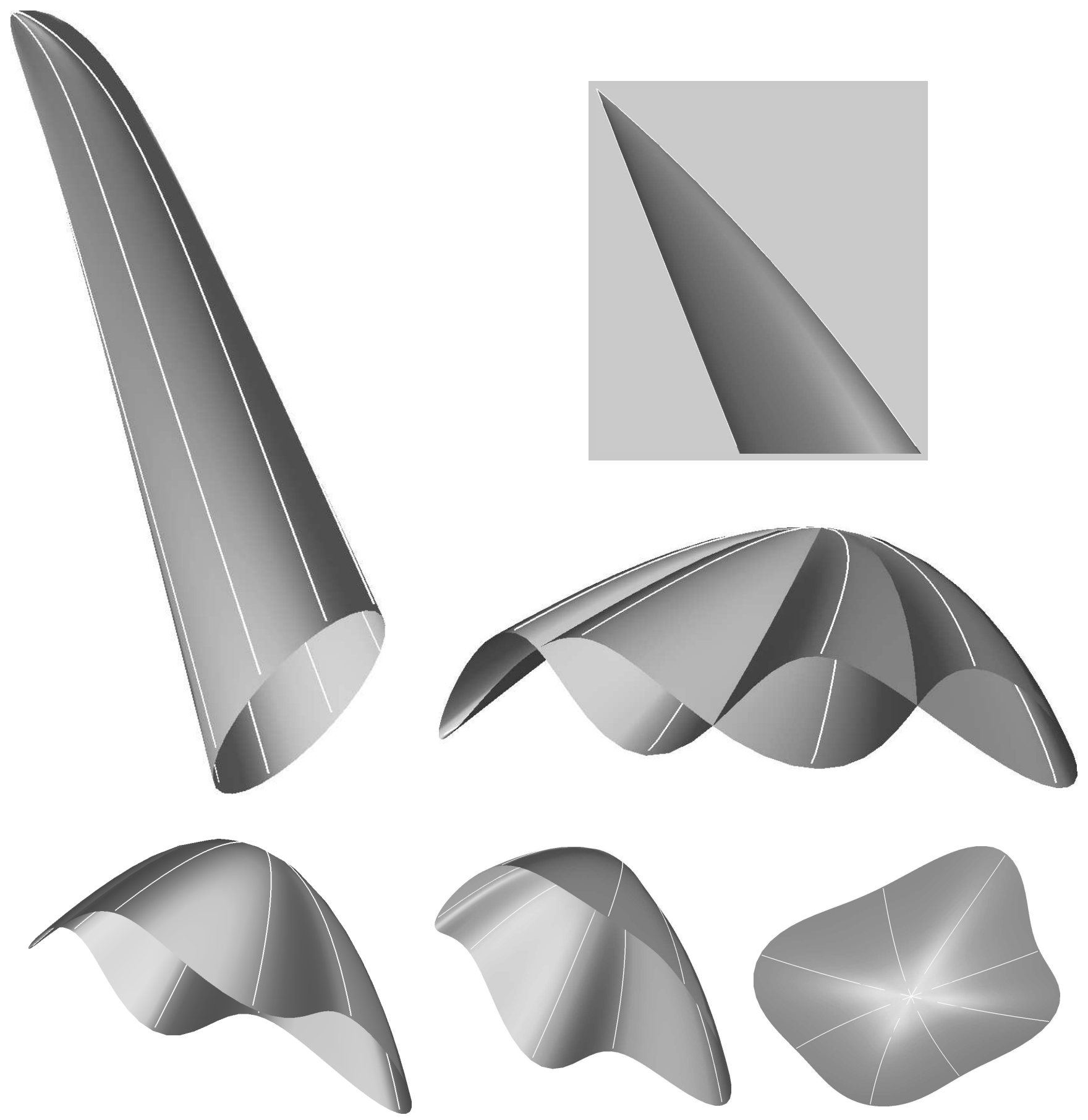

FIGURE 16. Several views of the resonance surface $\Gamma_{1 / 4}$. Top left: the whole surface. Right and bottom: a subset of the surface near the tip, shown in several projections (top right, projection to the parameter plane; bottom right, projection to the phase plane). 

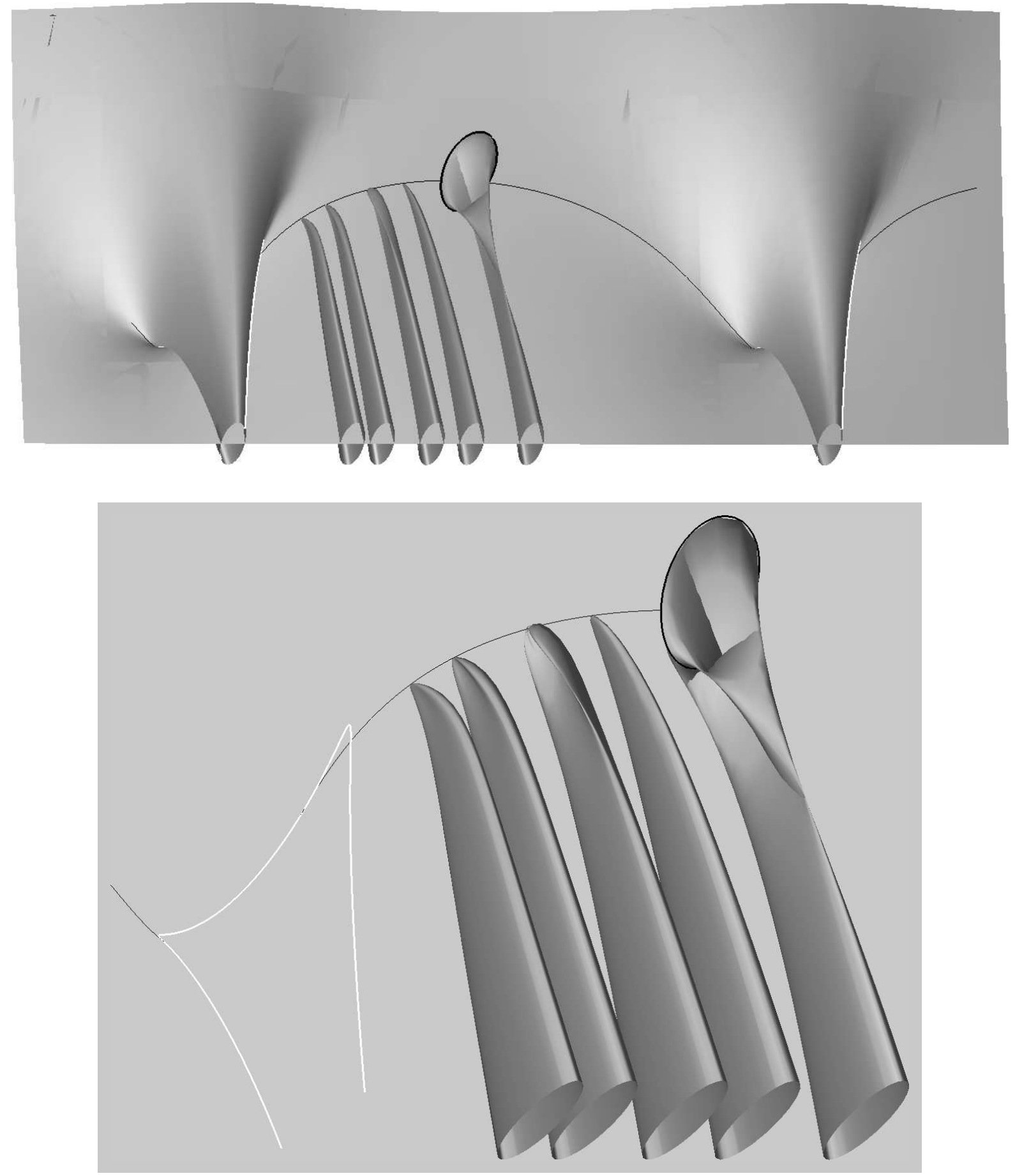

FIGURE 17. Ensemble of resonance surfaces. At the top we show $\Sigma(1)$ and $\bar{\Gamma}_{p / q}$ for $p / q=\frac{1}{5}, \frac{1}{4}, \frac{1}{3}, \frac{2}{5}, \frac{1}{2}$. At the bottom we have omitted the fixed-point surface $\Sigma(1)$. We have included the curves of saddle-node bifurcation, Hopf bifurcation, and period-doubling for reference (with the conventions of the table on page 232). When projected to the parameter plane, these curves revert to the more traditional (and more mundane) parameter space bifurcation diagram of Figure 1. 


\subsection{An Ensemble}

There is a resonance region and a corresponding resonance surface associated with every rational rotation number. The $p / q$ resonance region emanates from the point $(\beta, \alpha)=(p / q, 0)$ in the parameter plane and connects the $\beta$-axis to the curve of Hopf bifurcation. The corresponding resonance surface $\bar{\Gamma}_{p / q}$ has a boundary component consisting of the circle $C_{0}$ at the point $(\beta, \alpha)=(p / q, 0)$ and intersects the fixed point surface $\Sigma(1)$. For $q \geq 3$, this intersection occurs at a point of Hopf bifurcation. Figure 17 gives an idea of the whole.

\section{ACKNOWLEDGMENTS}

Bruce Peckham thanks the Geometry Center for its support and hospitality during his visit in the fall of 1992. Both authors acknowledge the use of Geometry Center facilities and acknowledge with gratitude the help of the Geometry Center staff.

\section{REFERENCES}

[Arnol'd 1982] V. I. Arnol'd, Geometric Methods in the Theory of Ordinary Differential Equations, Springer, New York, 1982.

[Aronson et al. 1982] D. Aronson, M. Chory, G. Hall, and R. McGehee, "Bifurcations from an invariant circle for two-parameter families of maps of the plane: a computer-assisted study", Comm. Math. Phys. 83 (1982), 304-354.

[Aronson et al. 1986] D. Aronson, R. McGehee, I. Kevrekidis, and R. Aris, "Entrainment regions for periodically forced oscillators", Phys. Rev. A33 (1986), 2190-2192.

[Bogdanov 1976] R. I. Bogdanov, "A versal deformation of a singular point of a vector field in the plane in the case of vanishing eigenvalues" (in Russian), Trudy Seminara Imeni I. G. Petrovskogo 2 (1976), 37-65. English translation, Selecta Math. Sovietica 1 (1981), 373-388.
[Chenciner 1985] A. Chenciner, "Bifurcation de points fixes elliptiques. II. Orbites periodiques et ensembles de Cantor invariants", Inv. Math. 80 (1985), 81-106.

[Frouzakis et al. 1991] C. E. Frouzakis, R. A. Adomaitis and I. G. Kevrekidis, "Resonance phenomena in an adaptivelly-controlled system", Int. J. Bifurcations and Chaos, 1 (1991), 83-106.

[Guckenheimer and Holmes 1983] J. Guckenheimer and P. Holmes, Nonlinear Oscillations, Dynamical Systems, and Bifurcations of Vector Fields, Springer, New York, 1983.

[Hall 1984] G. R. Hall, "Resonance Zones in twoparameter families of circle homeomorphisms", SIAM J. Math. Anal. 15 (1984), 1075-1081.

[Kai and Tomita 1979] T. Kai and K. Tomita, "Stroboscopic phase portrait of a forced nonlinear oscillator", Progr. Theor. Phys. 61 (1979), 54-73.

[Kevrekidis et al. 1986] I. G. Kevrekidis, R. Aris, and L. D. Schmidt, "The stirred tank forced", Chem. Eng. Sci. 41 (1986), 1549-1560.

[McGehee and Peckham 1992] R. P. McGehee and B. B. Peckham, "Resonance City" (10-minute video), The Geometry Center, University of Minnesota, 1992.

[McGehee and Peckham 1995] R. P. McGehee and B. B. Peckham, "Determining the Global Topology of Resonance Surfaces for Periodically Forced Oscillator Families", to appear in Proceedings of the Workshop on Normal Forms and Homoclinic Chaos, Fields Institute Communications Series, Amer. Math. Soc., Providence (RI), 1995.

[Peckham 1988] B. Peckham, "The closing of resonance horns for periodically forced oscillators", Ph.D. Thesis, University of Minnnesota, 1988.

[Peckham 1990] B. Peckham, "The necessity of the Hopf bifurcation for periodically forced oscillators", Nonlinearity 3 (1990), 261-280.

[Peckham and Kevrekidis 1991] B. Peckham and I. Kevrekidis, "Period doubling with higher order degeneracies", SIAM J. Math. Anal. 22 (1991), 1552-1574.

[Phillips et al. 1993] M. Phillips, S. Levy and T. Munzner, "Geomview: An Interactive Geometry Viewer", Notices of the Amer. Math. Soc. 40 (1993), 985-988. 
This software and the accompanying manual are avialable by anonymous ftp from the host geom. umn . edu, in directory pub/software.

[Schrieber et al. 1988] I. Schreiber, M. Dolnik, P. Choc and M. Marek, "Resonance behaviour in twoparameter families of periodically forced oscillators", Phys. Let. A128 (1988), 66-70.
[Takens 1974] F. Takens, "Forced oscillations and bifurcations", Comm. Math. Inst. Rijksuniversiteit Utrecht 3 (1974), 1-59.

[Vance and Ross 1989] W. Vance and J. Ross, "A detailed study of a forced chemical oscillator: Arnold tongues and bifurcation sets", J. Chem. Phys. 91 (1989), 7654-7670.

Richard P. McGehee, School of Mathematics, University of Minnesota, Minneapolis, Minnesota 55455 (mcgehee@math.umn.edu)

Bruce B. Peckham, Department of Mathematics and Statistics, University of Minnesota, Duluth, Minnesota 55812 (bpeckha1@arnold.d.umn.edu)

Received April 7, 1994; accepted in revised form November 28 